\title{
Improved detection of faint streaks based on a streak-like spatial filter
}

\author{
A. Vananti*, K. Schild, T. Schildknecht \\ Astronomical Institute, University of Bern, Sidlerstrasse 5, 3012 Bern, Switzerland \\ *Corresponding author, E-mail address: alessandro.vananti@aiub.unibe.ch
}

Keywords: streak detection, detection algorithm, image processing, space debris

\begin{abstract}
This paper presents an algorithm to detect very faint object streaks on CCD images acquired with an optical system. The proposed detection method uses image filters simulating the geometrical form and orientation of possible streaks on the CCD image. The method searches for a matching between streak and filter evaluating the convolutions of the image with all possible filters. Based on the statistics of the image background a threshold is applied in order to accept, respectively reject, the possible streak candidates. The detection probabilities and the effect of different parameter settings are estimated with tests on simulated images, while subframes of real images are used to evaluate the applicability of the algorithm to real cases. The detection probability of this method depends on the length and on the signal-to-noise ratio of the streak. For long streaks, a detection for signal-to-noise values around 0.5 is achieved. The further characterization of the detected streak (e.g. centroid and length), which is not performed in the current algorithm, and the reduction of the computation time, which is relatively high for full acquired frames, as well as possible improvements are briefly addressed.
\end{abstract}

\section{Introduction}

The interest in observing artificial objects in space started practically simultaneously with the launch of the first artificial satellite (Sputnik, 1957). Early methods of image analysis used strategies and criteria based on human eye inspection. With the digital era and the growing data amount, a trend is visible towards methods using greater computational effort and aiming at a higher degree of automation. Moreover, the need for detection systems with higher sensitivity has increased.

All detection methods found in today's literature try to extract the signal on the image related to the space object and to distinguish the latter from the image background. There are various approaches to extract the relevant signal from the image. The Stacking Method (Yanagisawa et al., 2003) collects the object signal by stacking subimages of subsequent short exposure CCD frames to sum the values of the pixels of interest. In the 3D Line Method (Yanagisawa et al., 2012) a series of short exposure images is taken and arranged in the x-y-t space. The bright pixels lying on a straight line, i.e. indicating a constant movement over time, are searched. Similar methods are described by other authors (Sara et al., 2013; Dao et al., 2013). Some methods use image rotation and median determination along the image rows to collect the information belonging to one streak (Yanagisawa \& Nakajima, 2005; Sustr, 2013). In (Levesque, 2009) a method for tracking satellites is proposed using an iterative convolution with matching filters of the expected length and orientation given by the known parameters of the satellite. Other methods apply the Radon transformation to subimages of a CCD frame in order to find image regions with higher intensity (Zimmer et al., 2013; Ciurte \& Danescu, 2014).

In those different detection methods, the distinction of the relevant signal from the background is usually characterized by a threshold justified considering the image background statistics. However, the place of the distinction criterion in the detection procedure can vary significantly and many algorithms use additional acceptance/rejection criteria.

Naturally, every method has its advantages and disadvantages. Stacking methods show an almost unbeatable detection sensitivity, unfortunately at the cost of an extraordinary computational effort. Line methods are fast if the sensitivity is reduced but the computation time increases exponentially with the number of candidates to be connected with lines. Image rotations and medians are very time consuming operations if they have to be done in large number. The methods based on Radon or similar transformations, although simple and fast, are not able to detect very faint streaks. In general for all methods, having more sensitivity means higher computation time and lower reliability (higher false alarm rate). 
In this paper a detection method is proposed where the signal of the object on the image is improved using a spatial filter that simulates the geometrical form of the object to be detected. Specifically, since we assume that the space object appears like a streak in the acquired image, a streak-like spatial filter is adopted. The proposed approach is similar to the one in (Levesque, 2009) mentioned before, but in the present algorithm streak matching filters with all possible orientations and lengths are applied to the image. While in the stacking methods the pixels of interest from different images are collected, in our method they are collected by extending and moving the collecting area through a spatial filter, which involves a less demanding process at the cost of a slightly lower signal-to-noise ratio of the streak. The result of the filtering process provides delimited regions of interest, in which the exact characteristics of the streak need to be extracted with further processing. However, the analysis in this paper is limited to the pure detection of the streak and does not cover the problem of finding the centroid or the exact shape and orientation of the streak, nor addresses the requirements and implications in the case of a fully automated detection tool. For the latter refer e.g. to (Virtanen et al., 2016; Torteeka et al., 2019). For a preliminary analysis of its applicability to real cases the detection method is applied to subframes with reduced size of real acquired CCD images.

\section{Proposed detection method}

\subsection{Basic detection method}

The main idea of the method is based on a simple spatial filtering approach. In order to collect the most information from the image region illuminated by the object of interest, filters matching the expected shape of this region are considered. The point spread function (PSF) can be assumed to be Gaussian. So an object on the frame is expected to show up as a Gaussian distribution around a point or as a streak, which is an integrated PSF along the line of movement. In this method the image is convolved with streak-like filters of different lengths and orientations. The statistics of the background is then used to accept or reject the filter matching, and thus the object region, according to the values obtained from the convolution, in the following also referred as convolution "response".

\subsubsection{Streak-like filter}

A streak-like filter tries to imitate the integrated PSF profile along the streak. In the implemented filter the integrated PSF is adopted with the only difference that all pixel values lower than $1 \%$ of the maximal pixel are set to zero. Fig. 1 shows an example of such a filter. To each pixel of the filter a value $a_{i}$ is assigned, normalized in such a way that $\sum_{i=1}^{n} a_{i}=1$.

All filters (different lengths and angles) can be prepared in advance. The PSF to create the filters can be matched to the image acquisition parameters, like pixel size, angular resolution and seeing. For the examples and tests that follow a FWHM of $2 \mathrm{px}$ for the PSF is assumed.

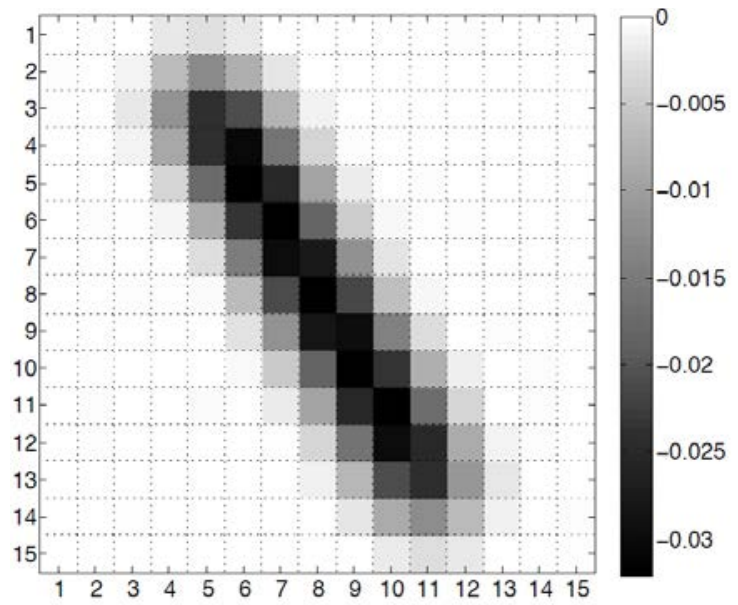

Fig. 1. Example of filter. Length $L=13 \mathrm{px}$, angle $=30^{\circ}$. The pixel values $a_{i}$ are normalized such that $\sum_{i=1}^{n} a_{i}=1$.

\subsubsection{Image background}


The background can be assumed to be constant and homogeneous in a local neighbourhood. Its pixel values $\mathrm{X}$ are in a good approximation normally distributed $\left(X \sim N\left(x, \mu_{0}, \sigma_{0}\right)\right)$ with

$$
\mu_{0}=\lambda_{B G}+\lambda_{D} \quad \text { and } \quad \sigma_{0}=\sqrt{\lambda_{B G}+\lambda_{D}+\sigma_{R}^{2}},
$$

where:

$$
\begin{aligned}
& \lambda_{B G}=\text { background signal value, } \\
& \lambda_{D}=\text { dark current signal value, } \\
& \sigma_{R}=\text { readout noise. }
\end{aligned}
$$

On pure background regions with known $\mu_{0}$ and $\sigma_{0}$, it is now possible to model the distribution of the convolution response $Y$ of any filter matrix containing the values $a_{1}, a_{2}, \ldots, a_{n}$ :

with $a_{i} \in \mathbb{R}$ and $X_{1}, \ldots, X_{n} \sim N\left(\mu_{0}, \sigma_{0}\right)$. It follows that:

$$
Y=\sum_{i=1}^{n} a_{i} X_{i}
$$

$$
\mu=\mu_{0} \quad \text { and } \quad \sigma=K \sigma_{0},
$$

where

$$
K^{2}=\sum_{i=1}^{n} a_{i}^{2}
$$

and $Y \sim N(\mu, \sigma)$.

\subsubsection{Acceptance criterion}

We define the overall acceptance threshold as $\mu+b \sigma$. If we choose e.g. $b=5$ a convolution value above the $5 \sigma$ threshold belongs only with a probability of $3 \cdot 10^{-7}$ to a pure background distribution. In order to characterize the intensity of a streak, we define the maximal pixel signal-to-noise ratio $(M t N)$ by:

$$
M t N=N_{O b j}(x, y) / \sigma_{0}(x, y),
$$

where $(x, y)=\arg \max _{x^{\prime}, y^{\prime}} N_{O b j}\left(x^{\prime}, y^{\prime}\right)$. The total noise of the pixel $(x, y)$ is given by $\sigma_{0}(x, y)$ and $(x, y)$ denotes the pixel where the number of object photons $N_{O b j}$ is maximal. The $M t N$ can especially be used for simulated streaks since in this case the object contribution is well known. Fig. 2 illustrates the main idea of this detection approach. The relative positions of the convolution response distributions for pure background and streaks are shown for different streak lengths. Note that, although the widths of the two distributions seem to be equal, in general the streak distribution contains the Poisson noise of the streak itself and can slightly differ. Obviously, with increasing streak lengths $L$ and the same $M t N$, the detection probability of the method increases as well. 

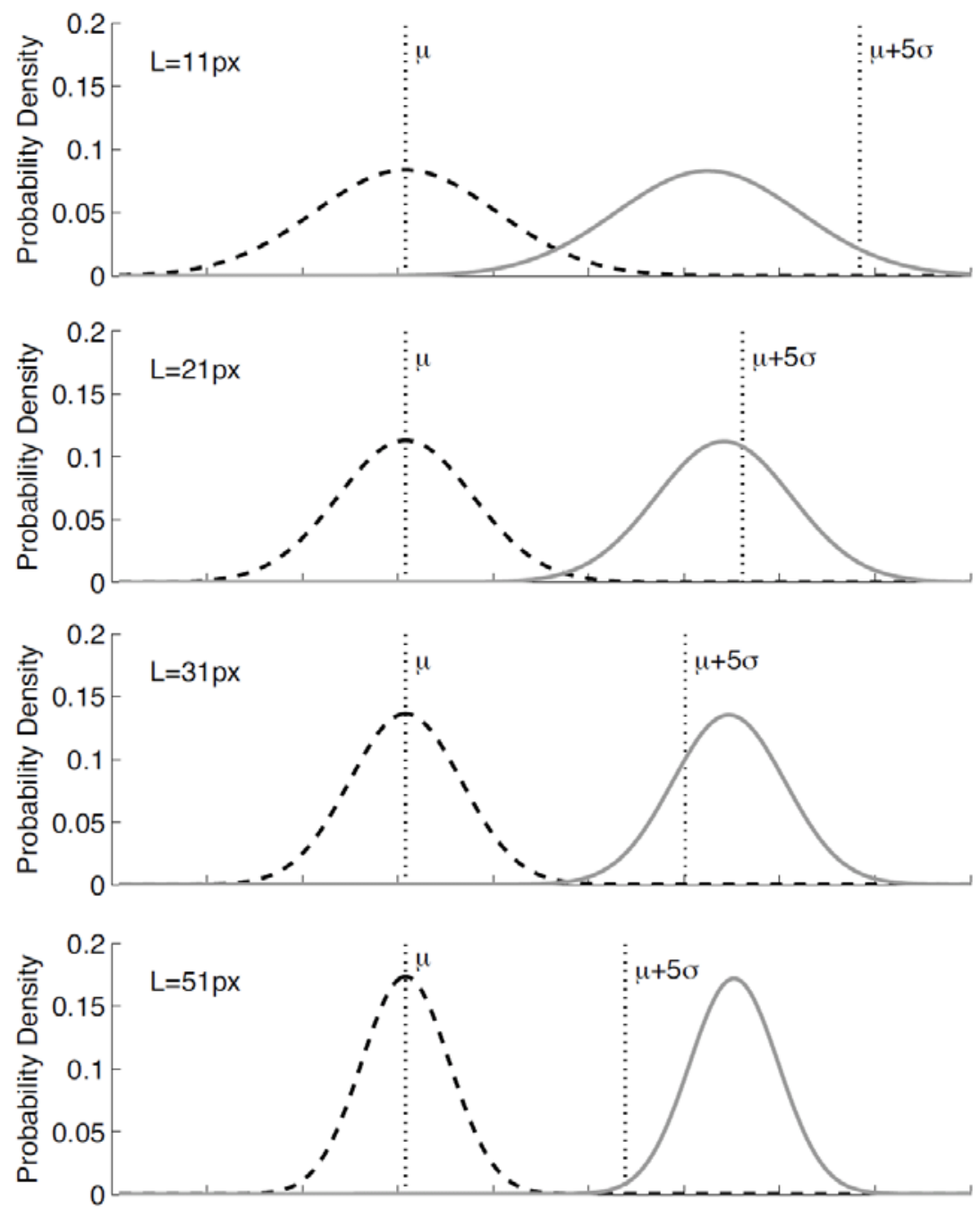

Fig. 2. Comparison of convolution response distributions for pure background and for streaks of different lengths $L$. The Maximum to Noise ratio $(M t N)$ is always 0.8. Broken line: modeled distribution of pure background (equations (3) and (4)). Continuous line: distribution estimated from 10’000 means determined by a filter perfectly matching a simulated streak. Fine dotted lines: threshold at $\mu+5 \sigma$.

\subsubsection{Structure of the algorithm}

A set $\Lambda$ of possible filter lengths and a set $A$ of filter orientations are assumed. See section 3.2 and 3.3 for details about the optimal sets $\Lambda$ and $A$. The pixel values of the image $I$ are defined as $I(x, y)$ where $x=1,2, \ldots, n$, and $y=1,2, \ldots, m$. The algorithm loops over all the lengths $L \in \Lambda$ and angles $\alpha \in A$ and computes the convolution $C(x, y, L, \alpha)$ of the image $I$ with the filter function $F(x, y, L, \alpha)$ at all coordinates $x, y$. The values of $C$ are expressed in the following as number of $\sigma$ above $\mu$ of the background. The angle $\alpha_{\max }$ corresponding to the maximum value of $C$ with given $L$ is determined for each pixel:

$$
\alpha_{\max }(x, y, L)=\arg \max _{\alpha} C(x, y, L, \alpha) .
$$

The $C\left(x, y, L, \alpha_{\max }\right)$ and $F\left(x, y, L, \alpha_{\max }\right)$ values over all the lengths $L$ which satisfy a certain threshold condition are summed up and give $S_{C}$ and $S_{F}$ :

$$
\begin{aligned}
& S_{C}(x, y)=\sum_{L \in G} C\left(x, y, L, \alpha_{\max }\right) \\
& S_{F}(x, y)=\sum_{L \in G} F\left(x, y, L, \alpha_{\text {max }}\right),
\end{aligned}
$$

where $G=\left\{L: C\left(x, y, L, \alpha_{\max }\right)>\mu+b \sigma\right\}$. 
In the end, $S_{C}$ contains the summation over all the accepted convolution values and indicates the centers of the accepted filter functions $F$ while $S_{F}$ contains all accepted filter functions $F$ of the whole procedure and indicates their geometry. Fig. 3 illustrates the principle of the method for one situation, namely for a streak's central pixel in the loop with a filter of the same length as the streak. Fig. 4 shows a simulated image and the resulting sets $S_{C}$ and $S_{F}$.
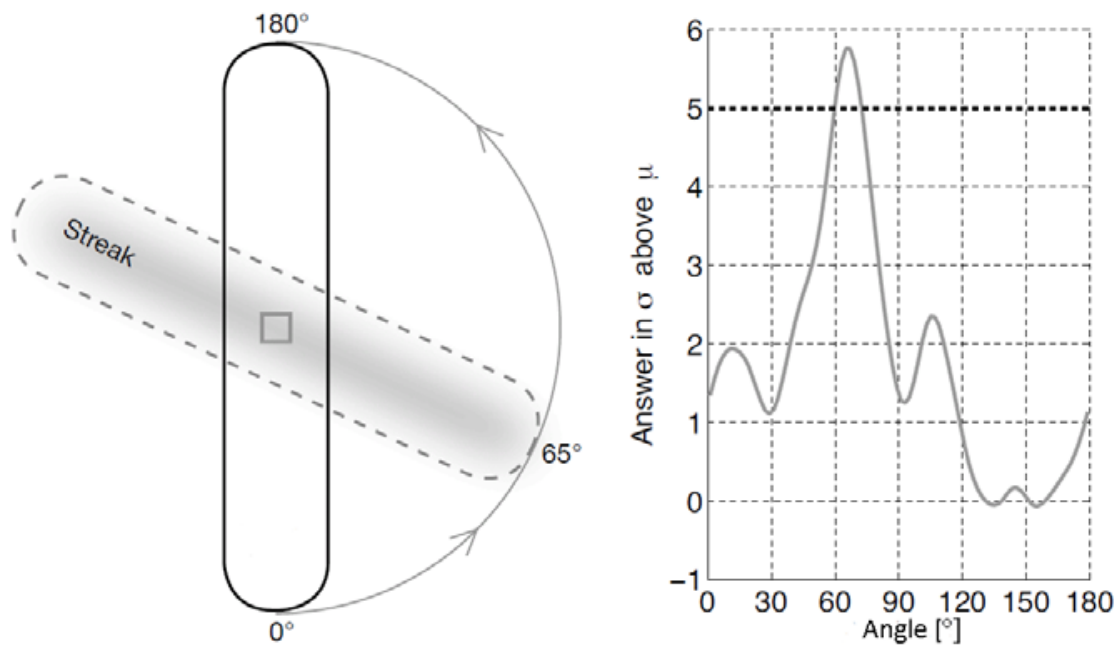

Fig. 3. Principle of the method for a filter of the same length of the streak. The right plot shows the convolution values $C(\alpha)$ (referred as convolution "answer") for the central pixel expressed in number of $\sigma$ above $\mu$. The dotted line indicates the $\mu+$ $5 \sigma$ threshold.
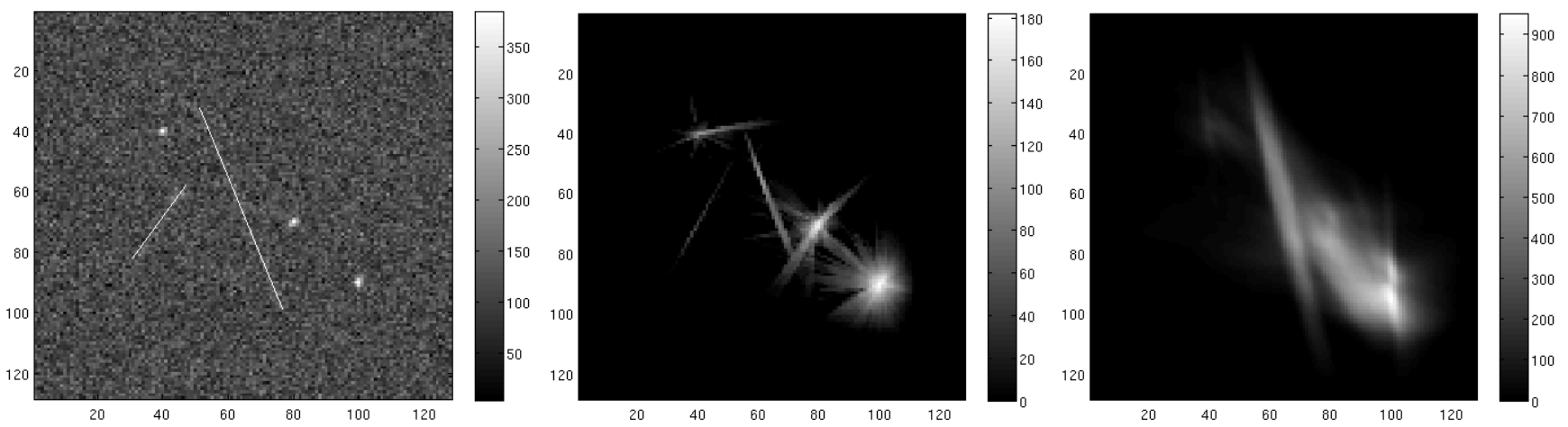

Fig. 4. Simulated image (left) and resulting set $S_{C}$ (middle) and $S_{F}$ (right). The simulated image contains three bright pointlike objects with $M t N=6,8$, and 10 (from the left) and two barely visible streaks with $M t N=1$ and 0.7 (from the left).

Two thin white lines indicate the approximate position of the streaks.

\subsection{Improving procedures}

Using the proposed baseline algorithm there are several artefacts that do not allow a perfect detection. Undesired detections come e.g. from partial covering of a second object which smears or even overlays the valuable information in the outputs $S_{C}$ and $S_{F}$. The main challenge is to avoid most of these undesired detections without losing too much sensitivity. The most typical problems are (Fig. 5):

Problem 1. Pixels surrounding a relatively bright object cause false alarms. This problem smears regions around objects: the object's shape itself is not well defined and possible faint objects in the same region are lost.

Problem 2. The maximum of the convolution response is obtained with the wrong filter orientation if the filter overlays nearby bright objects.

Problem 3. For relatively bright object the convolution response is accepted even if the length of the filter exceeds the length of the object. In extreme cases the length can reach multiples of the object's size. 


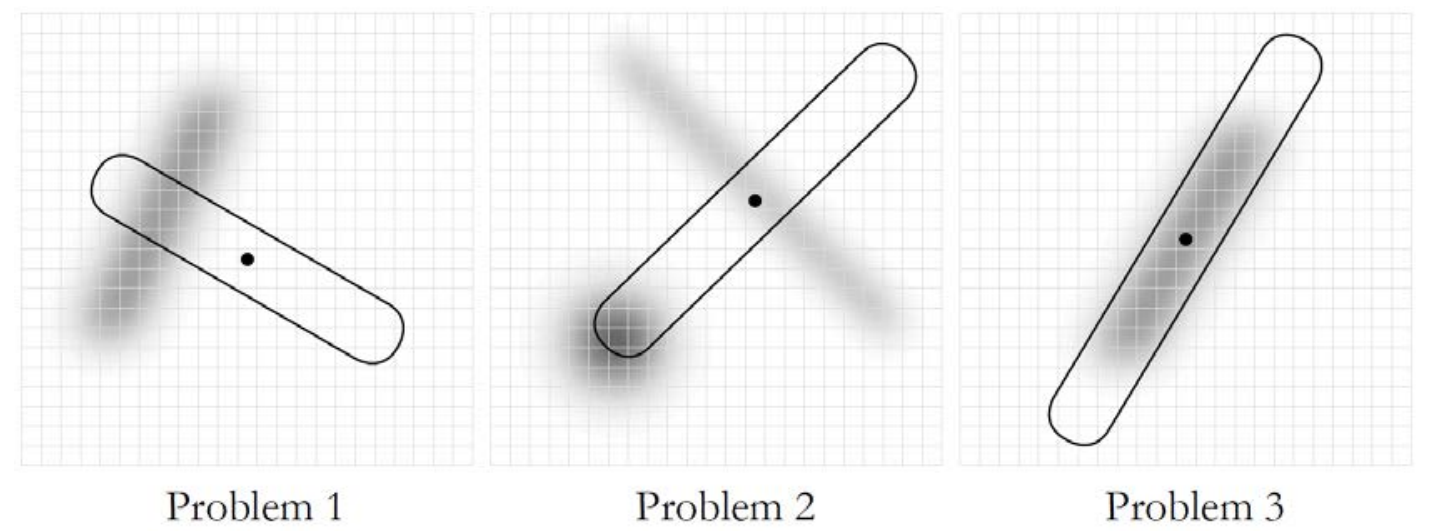

Fig. 5. Illustration of three typical problems.

To solve the mentioned problem additional procedures were developed to use the consecutive information resulting from the convolution during the iteration with filters of increasing lengths. It has to be pointed out that any additional criterion has the effect to slightly reduce the detection sensitivity. The following procedures show the best compromise to reduce false alarms but still guarantee a relatively good sensitivity.

\subsubsection{Maximum of accumulated convolution response (mitigation of Problem 2)}

The baseline method relies on convolutions with filters of increasing length $L$. The convolution response for filters up to the streak length is maximal around the same angle. For a more refined maximum criterion, instead of detecting the maximum of $C$, we determine the angle where the accumulated convolution response

$$
S_{L}(x, y, L, \alpha)=\sum_{\ell<L} C(x, y, \ell, \alpha)
$$

is maximal:

$$
\alpha_{\max }(x, y, L)=\arg \max _{\alpha} S_{L}(x, y, L, \alpha) .
$$

If $C\left(\alpha_{\max }\right)$ is above the $5 \sigma$ threshold the response and its corresponding filter function $F$ are accepted and included in the calculation of $S_{C}$ respectively $S_{F}$.

This correction solves the wrong orientation problem (Problem 2) to a high degree. The orientation of the streak is more emphasized.

\subsubsection{Upper clipping (mitigation of problem 1)}

Many objects are brighter than needed for a response above the $5 \sigma$ threshold. Brighter objects have a higher probability that surroundings or partially matching filters cause false alarms. If the image is clipped at an appropriate value, the detection of streaks is guaranteed while the probability for false alarms can be reduced. Upper clipping of the image $I$ at a value $c$ is defined as:

$$
\begin{array}{ll}
I(x, y)=c, & \text { if } I(x, y) \geq c \\
I(x, y)=I(x, y), & \text { if } I(x, y)<c .
\end{array}
$$

The value $c$ is a function of the length and has to be chosen with caution in order not to lose detection sensitivity. In case of high spatial variability of the noise across the image (e.g. strongly vignetted images) the value $c$ may be dependent from the position on the frame. In the following we will indicate the clipping parameter as $c(L, x, y)$ but for simplicity we will not consider spatial variations. For each filter length $L$, there is an $M t N$ value of a streak of length $L$ that results in a detection probability very close to 1 . Thus it makes sense to clip the image just slightly above the expected maximal pixel values of such a streak. Fig. 6 illustrates this idea. Clipping is a powerful tool and reduces Problem 1 very effectively. 


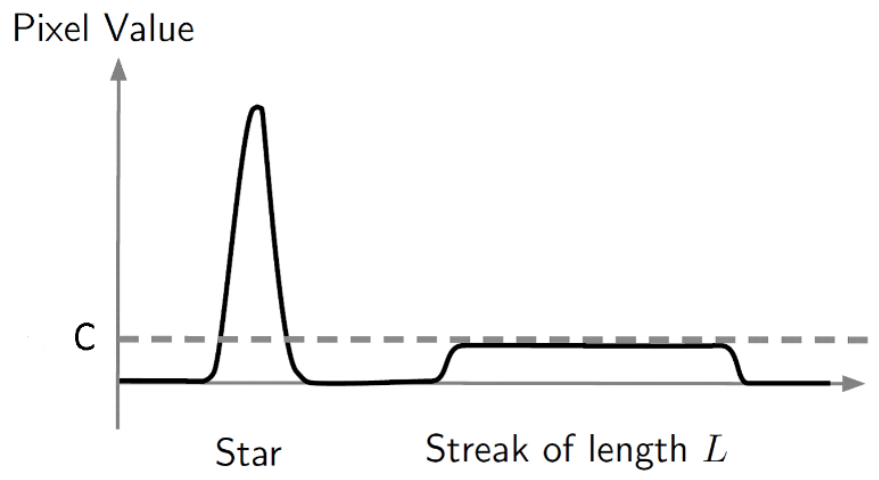

Fig. 6. Clipping parameter $c(L, x, y)$ for the convolution with the filter function $F(L)$. The streak has a brightness (resp. $M t N$ ) that leads to a detection probability $P=0.999$.

\subsubsection{Convolution response evolution (mitigation of Problem 1 and false positives)}

Looking at the evolution w.r.t. the length $L$ of the convolution response (values in $C$ ) along a certain angle $\alpha$ enables us to define some further criteria to distinguish between streak and non-streak regions. In Fig. 7, typical curves of the evolution of $C$ for three different cases are illustrated: streak (left), star (center), background (right). The following characteristics seem to occur with great probability:

(a) Streak: the $C$ values are expected to increase with growing length $L$. They reach a maximum around the streak length and then decrease slowly. Thus if the response surpasses the $5 \sigma$ threshold at some point, it might stay above this threshold even if $L$ is longer than the streak.

(b) Star: at short lengths, the response has background characteristics: low values, rising and falling. When the filter starts to cover the star there is a steep increase, possibly reaching the threshold. The following flattening of the trend depends on the extension of the object.

(c) Background: the response of background regions is distributed around zero. The values might rise or fall with growing length and there is not a steady increase as for streaks.

In order to weaken or even exclude uninteresting image regions and strengthen regions of interest, we define $P_{L}$ :

where

$$
P_{L}(x, y, L, \alpha)=\prod_{\ell<L} p_{C}(x, y, \ell, \alpha),
$$

$$
p_{C}(x, y, L, \alpha)=\operatorname{normcdf}(C(x, y, L, \alpha)) .
$$

At every length $L$ the convolution response $C$ is used to define a probability given by the cumulative distribution function. In $P_{L}$ the products of these probabilities up to the length $L$ are computed. Values close to one for clear object regions along the objects angle and values quickly going towards zero for background regions are expected. New criteria to accept respectively reject the convolution response are introduced.

Rule 1. $C$ values below a certain threshold $\gamma_{C}$ are excluded to avoid background regions being the center of a possible false alarm. $P_{L}$ is set according to the condition:

$$
P_{L}=0 \text {, if } C(L, \alpha) \leq \gamma_{C} .
$$

Rule 2. If the value of the function $C(L, \alpha)$ decreases by a certain amount $\gamma_{M}$ w.r.t. its maximum it is excluded. This rejects many background regions, and for streaks, it is meant to avoid further obsolete calculations if the length of the filter has surpassed the length of the streak. The maximum of the $C$ evolution is defined as $M_{C}$ and the following condition is applied:

$$
\begin{aligned}
& M_{C}(L, \alpha)=\max _{\ell<L} C(\ell, \alpha) \\
& P_{L}=0, \text { if } C(L, \alpha) \leq M_{C}(L, \alpha)-\gamma_{M} .
\end{aligned}
$$

Rule 3. $P_{L}$ values lower than a certain threshold $\gamma_{P}$ are excluded:

$$
P_{L}=0 \text {, if } P_{L} \leq \gamma_{P}
$$


Rule 4. A response over the $5 \sigma$ threshold is only accepted if it is greater than or equal to the response of the preceding length. This preserves most of the object detections and stops the acceptance if the $C$ values start to drop:

$$
C\left(L_{i}, \alpha\right) \geq C\left(L_{i-1}, \alpha\right) .
$$

With these additional rules, a response is accepted if it is above the $5 \sigma$ threshold, if the inequality (19) holds, and if the corresponding $P_{L}$ entry is greater than zero. Furthermore, in the final results the response is weighted by the $P_{L}$ value:

$$
\begin{aligned}
& S_{C}(x, y)=\sum_{L \in G} C\left(x, y, L, \alpha_{\text {max }}\right) P_{L}\left(x, y, L, \alpha_{\text {max }}\right) \\
& S_{F}(x, y)=\sum_{L \in G} F\left(x, y, L, \alpha_{\text {max }}\right) P_{L}\left(x, y, L, \alpha_{\text {max }}\right) .
\end{aligned}
$$

The criteria based on the response evolution reduce Problem 1. Contrary to expectations, Rule 2 does not efficiently solve Problem 3 . The decrease of the $C(L, \alpha)$ values is relatively slow and is responsible of an overestimation of the object length.
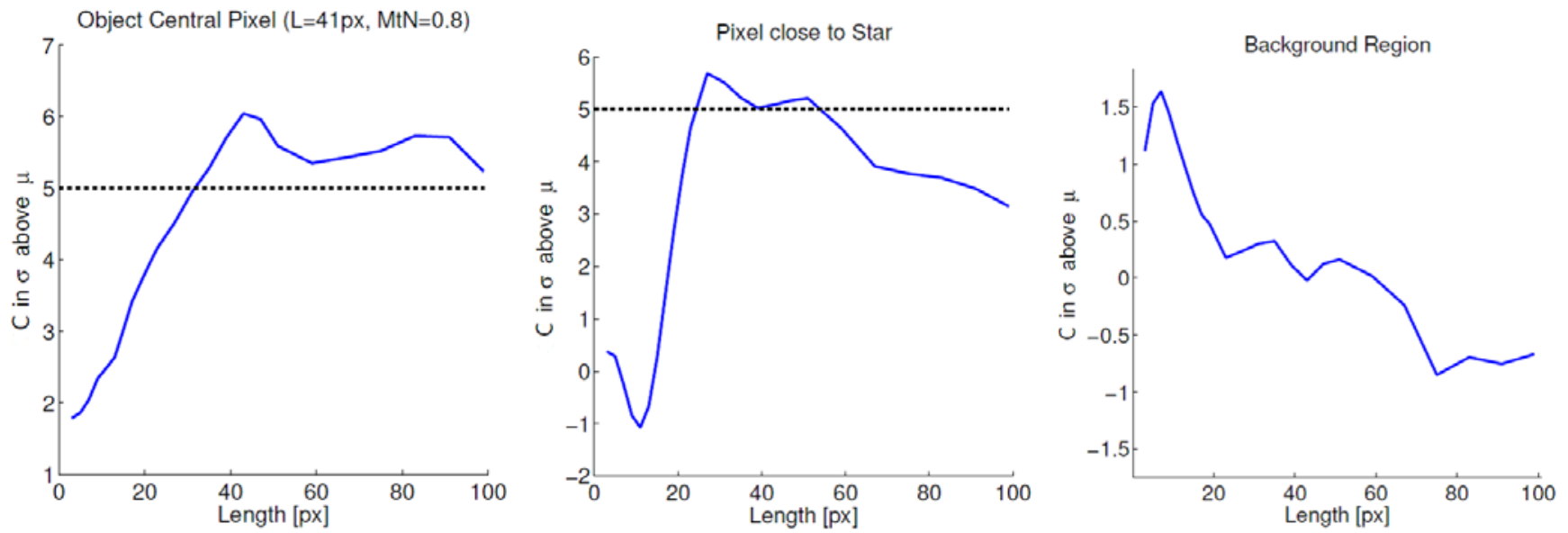

Fig. 7. Typical evolution of $C(\alpha)$ for different situations: (a) streak (left), (b) star (center), (c) background (right).

\subsubsection{Length limitation (mitigation of Problem 3)}

For a streak and filter of length $L$ the maximum of $S_{L}$ lies in the center of the streak and decreases towards the end points, where the value is around $50 \%$ of the maximum and the filter only covers half of the streak. An example of this effect is illustrated in Fig. 8 for a streak $40 \mathrm{px}$ long and $M t N=1.5$.

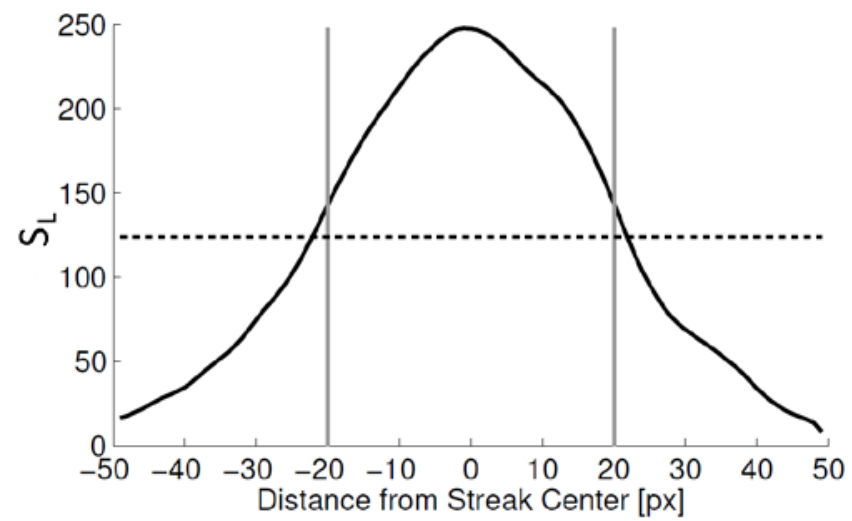

Fig. 8. Scanning of $S_{L}$ along the central line of a filter candidate. Grey vertical lines indicate the real length of the streak. Black dotted line is $50 \%$ of the maximal value.

This can be exploited to set a condition to better evaluate the length of the detected object. We can imagine an ideal line of length $L$ through the center of the filter and parallel to it. Then we consider the coordinates $x^{\prime}, y^{\prime}$ of the pixels touched by this ideal line. Since $x^{\prime}$ and $y^{\prime}$ are integer values they are rounded up in the equation of the ideal line. The latter is defined as: 


$$
R_{L, \alpha, x, y}=\left\{x^{\prime}, y^{\prime} \in \mathbb{N}: y^{\prime}=\left[q(\alpha)\left(x^{\prime}-x\right)+y\right],\left(x-x^{\prime}\right)^{2}+\left(y-y^{\prime}\right)^{2} \leq \frac{L^{2}}{4}\right\}
$$

where $q(\alpha)$ is the slope in the equation of the line depending on the angle $\alpha$, while an additional equation delimits the length $L$.

If all $S_{L}\left(x^{\prime}, y^{\prime}\right)$ values are greater than $50 \%$ of their maximum the response is accepted and the summation in the equations (7) and (8) goes over $G$ redefined as:

$$
G=\left\{L: \forall x^{\prime}, y^{\prime} \in R_{L, \alpha_{\max }, x, y}, S_{L}\left(x^{\prime}, y^{\prime}, L, \alpha_{\max }\right)>\frac{1}{2} \max _{x^{\prime}, y^{\prime} \in R_{L, \alpha_{\max }, x, y}} S_{L}\left(x^{\prime}, y^{\prime}, L, \alpha_{\max }\right)\right\} .
$$

This approach effectively solves Problem 3 and the streaks are better defined in length.

\subsubsection{Angle restriction (reducing computational effort)}

In general if the response of a streak region has already high values at short lengths then the resulting $P_{L}$ value stays around 1 for increasing lengths. This allows us to restrict the number of angles to be considered during the procedure keeping the same sensitivity. For a given $x$ and $y$ only the angles where $P_{L}$ is greater than a certain threshold $\gamma_{R}$ are further considered in the iteration over the increasing length $L$. The set of angles in (7) is reduced according to:

$$
A_{R}(x, y, L)=\left\{\alpha \in A: P_{L}(x, y, L, \alpha)>\gamma_{R}\right\} .
$$

For example, in Fig. 9 the reduction with $\gamma_{R}=0.995$ of the number of angles with increasing length is shown for a test image with the presence of a long streak (length $L=71 \mathrm{px}, M t N=0.7$ ) which is mainly characterizing the reduction trend. While the detection remains unchanged the computation time is only roughly $60 \%$ of the time needed without restriction.

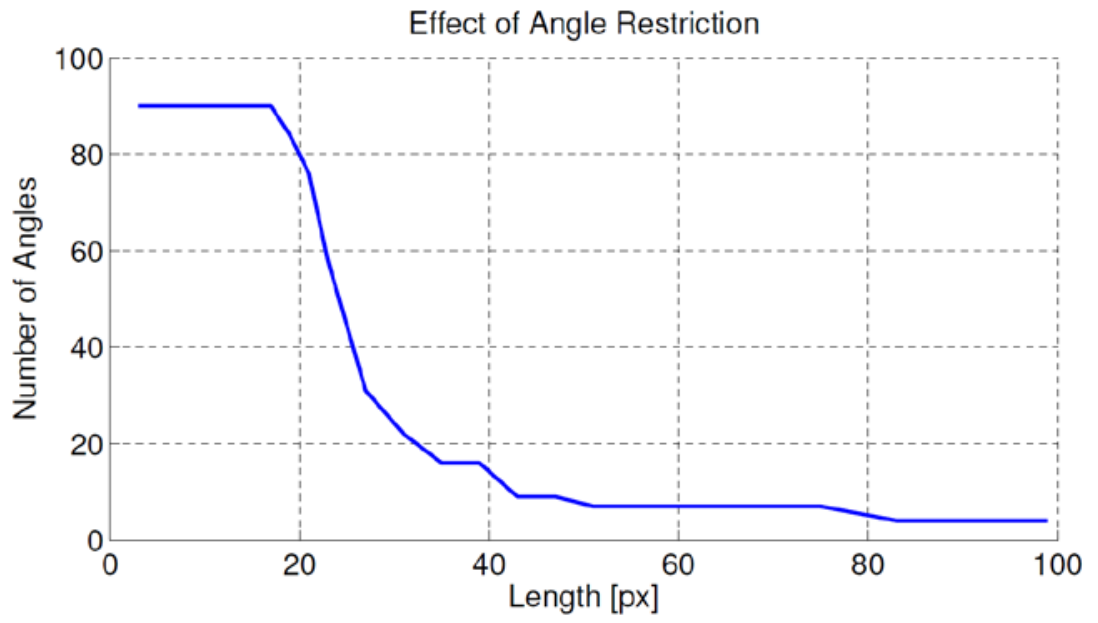

Fig. 9. Number of remaining angles with increasing length. Used threshold: $\gamma_{R}=0.995$.

\subsubsection{Summary of the algorithm parameters}

In Table 1 the parameters of the algorithm are summarized with the respective detection criteria and default values. For the justification of the default values see section 3. Every row represents a possible improvement procedure with respect to the baseline in the first row. Combinations of several procedures are possible. 


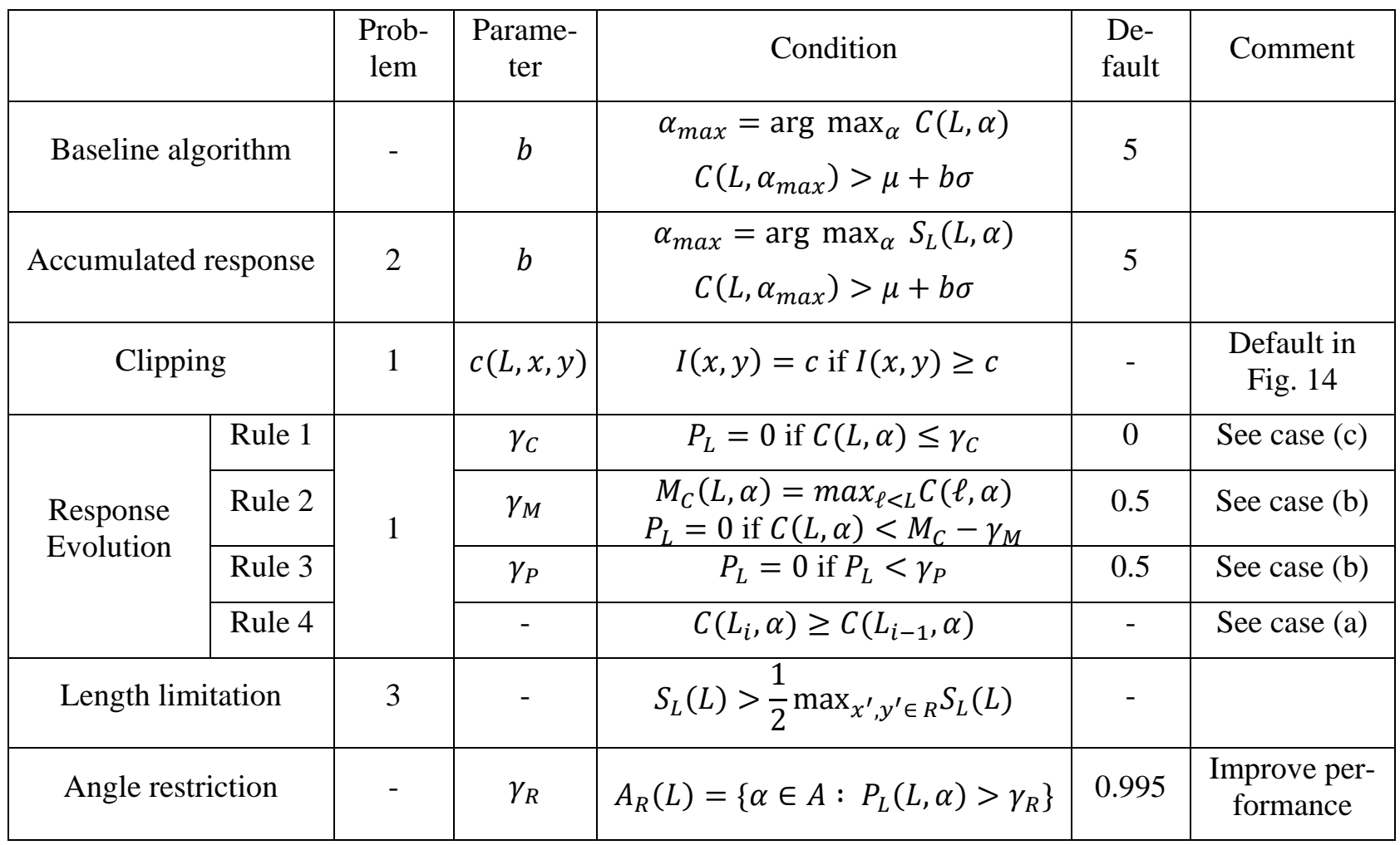

Table 1: List of algorithm parameters with detection criteria and default values.

\section{Choice of the optimal settings}

For the choice of the optimal settings different types of tests on simulated images have been performed. For all the tests, images containing one simulated streak of given $M t N$ value and length $L$, with an orientation of 90 degrees and with the center at a pixel center were generated. Tests with other orientations and positions within the pixel have shown only small differences from the one proposed. A background value $\mu=\lambda_{B G}+\lambda_{D} \approx 900$ ADU and $\sigma_{R}=5 \mathrm{e}$-/px were chosen as representative values. These values approximately correspond to the values present in the real frames acquired with the ZimSMART telescope (see section 4). As shown in section 3.1 the maximal pixel signal contribution can be expressed as a function of the $M t N$ value in order to simulate the object signal. For every test the same image was generated up to 1000 times with random noise. After applying the detection method, if the resulting set $\mathrm{S}_{\mathrm{C}}$ shows any non-zero value in the streak region, the streak is considered to be detected. The streak region is defined as the non-zero area of the filter perfectly matching the streak. Here a perfect match means that the filter has the same center, length and angle as the streak.

\subsection{Detection probability}

To derive a probabilistic model for the detection method including all the parameters would be very complex. But perfect match probabilities can be easily calculated and serve in a good approximation as an estimator. From the definition of the $M t N$ value

$$
M t N^{2}=S^{2} /\left(\lambda_{B G}+\lambda_{D}+\sigma_{R}^{2}+S\right)
$$

a general expression for the maximal pixel signal contribution $S$ can be derived:

$$
S=\frac{1}{2}\left(M t N^{2}+\sqrt{M t N^{4}+4 M t N^{2}\left(\lambda_{B G}+\lambda_{D}+\sigma_{R}^{2}\right)}\right) .
$$

For each pixel in the filter related to a matrix element $a_{i}$ we have:

$$
\mu_{0 i}(S)=\lambda_{B G}+\lambda_{D}+S \frac{a_{i}}{a_{\max }}
$$




$$
\sigma_{0 i}(S)=\sqrt{\lambda_{B G}+\lambda_{D}+\sigma_{R}^{2}+S \frac{a_{i}}{a_{\max }}}
$$

Then the distribution of the mean of the perfect match is a normal distribution with $\mu_{t o t}(S)=\sum_{i} a_{i} \mu_{0 i}(S)$ and $\sigma_{\text {tot }}(S)^{2}=\sum_{i} a_{i}^{2} \sigma_{0 i}(S)^{2}$. With the standard deviation $\sigma$ and mean $\mu$ coming from the pure background we define the threshold $\gamma$ as:

$$
\gamma=\mu+b \sigma
$$

Finally, the probability $P$ that a response $X$ is greater than $\gamma$ is computed by the normal cumulative distribution function normcdf() of the perfect match:

$$
P(X>\gamma)=1-\operatorname{normcdf}\left(\gamma, \mu_{\text {tot }}(S), \sigma_{\text {tot }}(S)\right) .
$$

Equation (30) enables us to estimate the detection probability of the method for different $M t N$ values. Fig. 10 illustrates just one such estimation and shows the detection probability for a streak of length $L=41 \mathrm{px}$ for different $M t N$ values depending on b varying between 5 and 7 . The choice of the parameter $b$ is usually a compromise between the number of correct detections and false alarms and there are no strict rules to define it. It depends on the requirements set by the user of the detection algorithm. According to the results shown in Fig. 10 we decided for a default threshold $b=5$, with a relative good detection of medium-length streaks with $M t N$ around 1 . For shorter streaks the detection probability rapidly decreases: e.g. for $M t N=1$ and a streak length around $10 \mathrm{px}$ the probability is about 0.1 .

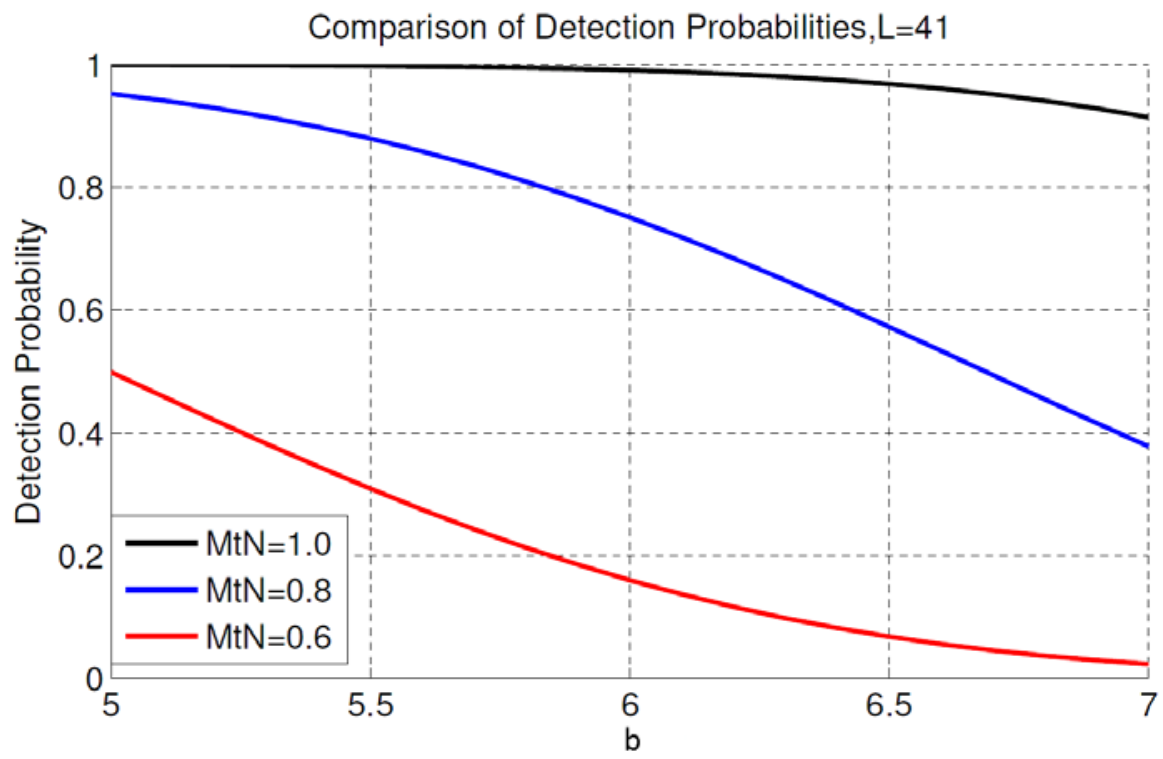

Fig. 10. Detection probability depending on the setting of $b$.

If the acceptance (given by the $5 \sigma$ threshold criterion) is not restricted by additional criteria, we expect for each length a theoretical false alarm probability $P_{F}$ given by the normal cumulative distribution function at a value of 5:

$$
P_{F}=1-\operatorname{normcdf}(5) \approx 2.87 \cdot 10^{-7}
$$

If $X(L)$ indicates the event of a false alarm in the convolution for the length $L$, then the total false alarm probability is calculated for all lengths $L \in \Lambda$, indicated as $L_{1}, L_{2}, \ldots, L_{n}$ :

$$
P_{F, \text { tot }}=P\left(X\left(L_{1}\right) \cup X\left(L_{2}\right) \cup \ldots \cup X\left(L_{n}\right)\right) .
$$

For any two probabilistic events $X$ and $Y$ we have:

$$
P(X \cup Y)=P(X)+P(Y)-P(X \cap Y) \text {. }
$$


Thus the resulting total probability is in general less than or equal to the sum of the probabilities of the individual events. By using the background statistics we can compute this probability step by step for all the lengths used in the method. The result for the total theoretical false alarm probability is $P_{F, t o t}=5.60 \cdot 10^{-6}$ or less. This theoretical probability does not take into account false alarms caused by field stars and their diffraction patterns, blended stars, cosmics, and other artefacts. The actual false alarm rate might be far from the theoretical value and has to be evaluated in the concrete case.

\subsection{Set of lengths $\Lambda$}

The length sequence in $\Lambda$ should be chosen in a way that the detection ability smoothly evolves with increasing length. To define the difference between the length $L_{i+1}$ and its preceding length $L_{i}$ we set the condition that, given the detection probability for a perfect match $P\left(L_{i+1}\right)>0.9995$, then the probability for $L_{i}$ is still $P\left(L_{i}\right)>0.999$. This defines the set of lengths in pixels $\Lambda=(3,5,7, \ldots, 23,27,31, \ldots, 51,59,67, \ldots, 99)$ shown in Fig. 11 as red points. For comparison also the conditions with $P\left(L_{i}\right)>0.99$ and $P\left(L_{i}\right)>0.95$ are shown.

Running the method with a reduced set of lengths would save a lot of computation time, but the determination of the length of the streak would not be that accurate any more. So at the present stage we would not recommend to use a reduced $\Lambda$. But there is definitely the possibility to adjust the length control process or to create a new process with equivalent performance such that a reduced set can be considered without a significant loss of detection probability.

The maximal considered length does not necessarily have to be 99 pixels. The choice depends on the expected maximal streak length. The filter can increase in length up to the dimension of the image if such a long streak is expected. However, in the latter case or in general with very long streaks there is a higher probability that the streak reaches the limit of the frame. In those cases the streak is still detected but it does not provide a reliable information about the position of the observed object.

A filter of a given length is able to detect also longer streaks but with a reduced probability. For relatively bright and very long streaks, many filters will overlap and show the extension of the streak very well. For very faint and very long streaks, the probability that any section of the streak reaches the threshold grows with increasing streak length.

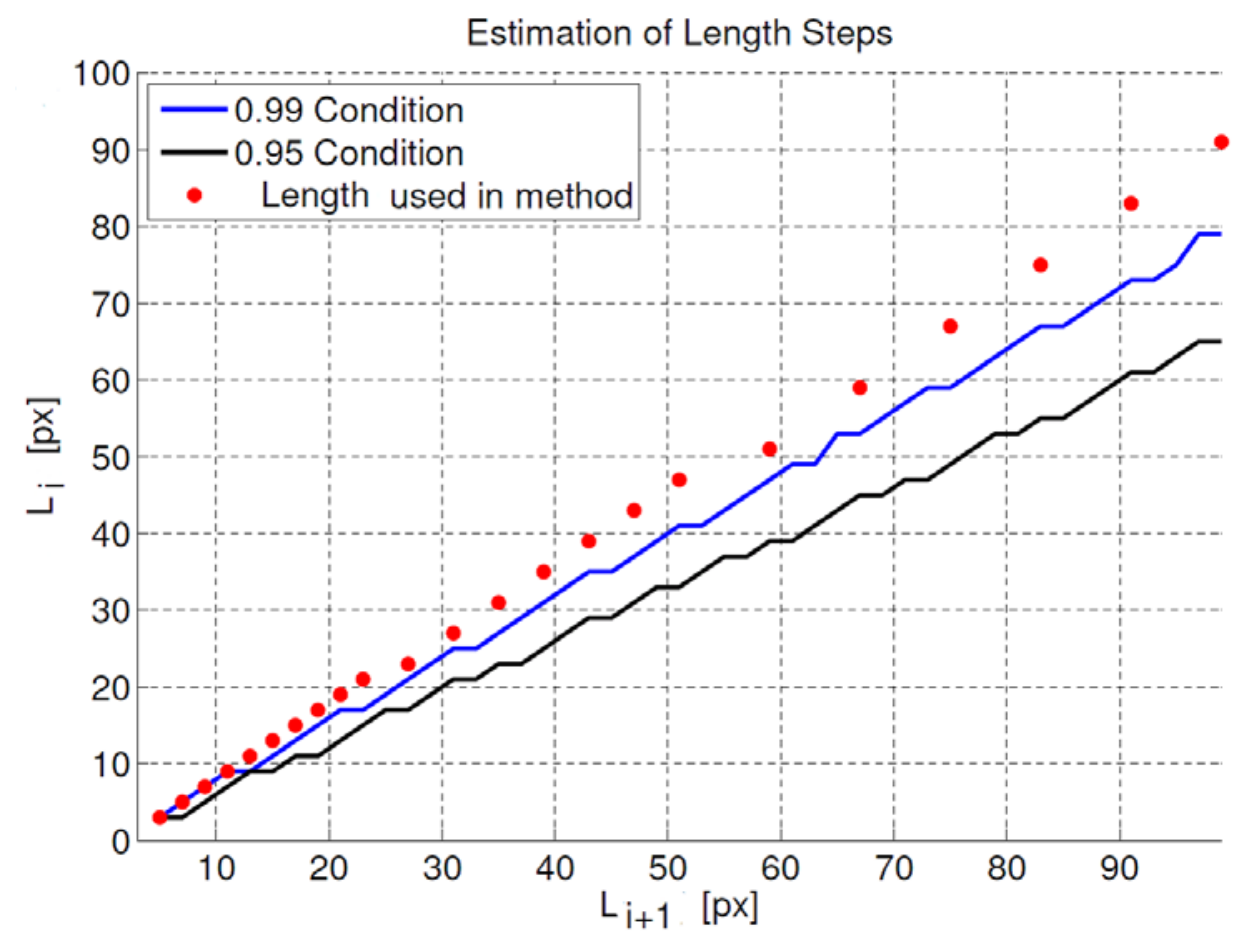

Fig. 11 Relation between a length $L_{i+1}$ and its preceding length $L_{i}$ when applying a certain probability relation condition. 


\subsection{Set of angles $A$}

In the choice of $A$ it is desired to have an angular resolution as good as possible. But this directly influences the computation time. Fig. 12 shows the modeled detection probability (equation (30)) for single matches with an angle difference of $\mathrm{d} \alpha$ between streak and filter. The results show that for $\mathrm{d} \alpha=1^{\circ}$ the loss in the detection probability is acceptable while for larger $d \alpha$ the loss gets more and more serious. Therefore a maximal angle difference $\mathrm{d} \alpha=1^{\circ}$ is chosen, which implies steps of $2^{\circ}$ in the definition of $A=\left(1^{\circ}, 3^{\circ}, 5^{\circ}, 7^{\circ}, \ldots, 179^{\circ}\right)$.

The set $A$ can be adapted for special tasks. For exposures e.g. in tracking mode the direction of the star motion in the frame is known. Or, if GEO objects are observed in sidereal tracking mode the streak direction on the frame lies within a certain range.

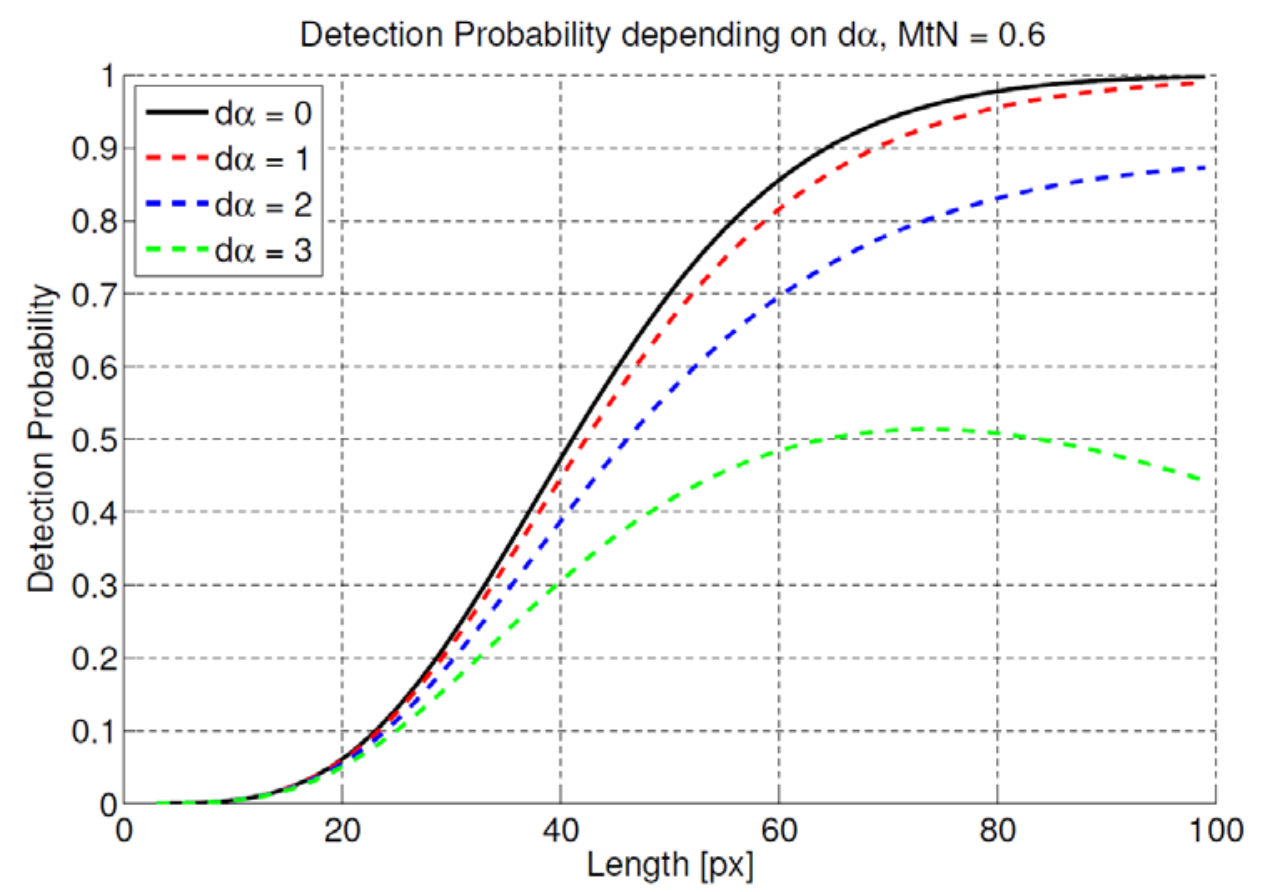

Fig. 12 Detection probability depending on the difference $\mathrm{d} \alpha\left[^{\circ}\right]$ between angle of streak and angle of filter $F$.

\subsection{Clipping parameter $c(L, x, y)$}

The clipping parameter $c(L, x, y)$ is chosen in such a way that the detection probability does not significantly decrease. For each length $L$ the $M t N$ value with a corresponding detection probability $P>0.999$ is determined. The results are illustrated in Fig. 13.

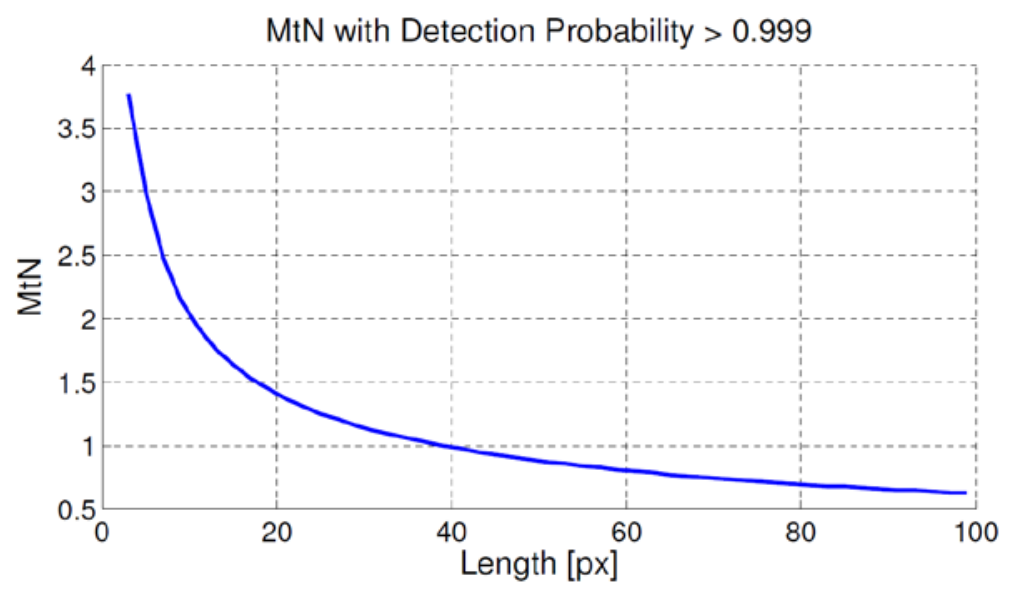

Fig. 13. $M t N$ with detection probability $P>0.999$ depending on length $L$. 
The statistics of the clipped case with mean $\mu^{\prime}$ and variance $\sigma^{\prime 2}$ can be calculated using the formalism for the truncated normal distribution (Johnson et al., 1994):

$$
\begin{aligned}
& \mu^{\prime}=\mu-\sigma \rho(k) \\
& \sigma^{\prime 2}=\sigma^{2}\left(1-k \rho(k)-\rho(k)^{2}\right),
\end{aligned}
$$

where $\rho(x)=N(x) / \operatorname{normcdf}(x), k=(c-\mu) / \sigma$, and $N(x)$ is the standard normal distribution.

To find the coefficient $k(L)$ the resulting detection probability is compared with the probability of the unclipped case. We determine the lowest $k(L)$ where $P>0.999$, which is the same condition of the unclipped case. The clipping coefficients resulting from these conditions are shown in Fig. 14. The clipping parameter $c(L, x, y)$ is then calculated from the clipping coefficient $k(L): c(L, x, y)=\mu+k(L) \sigma$.

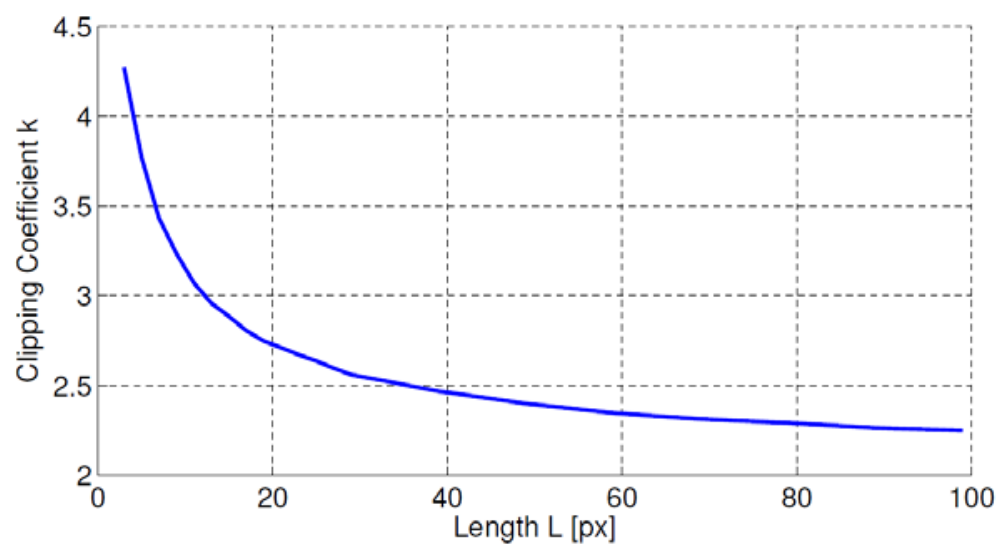

Fig. 14. Clipping coefficient $k(L)$ leading to $c(L, x, y)=\mu+k(L) \sigma$.

\subsection{Parameter $\gamma_{C}$}

The pixels with a convolution response lower than $\gamma_{C}$ are excluded from further investigation. Since low $C$ values in streak regions appear at short lengths we estimate the detection probability with equation (30) for a length of $L=3 \mathrm{px}$ (the smallest value in $\Lambda$ ) and for different $M t N$ values between 0.3 and 1.2. The probability that the response is greater than $\gamma_{C}$ is determined and the results are illustrated in Fig. 15. We see that down to an $M t N$ of approximately 0.4 , a setting of $\gamma_{C}=0$ will preserve $80 \%$ or more of all short streak sections $(L=3 \mathrm{px})$ and therefore is used as default setting. Since at this $M t N$ level only long streaks are detectable, the probability of preserving any response on such a streak is very high.

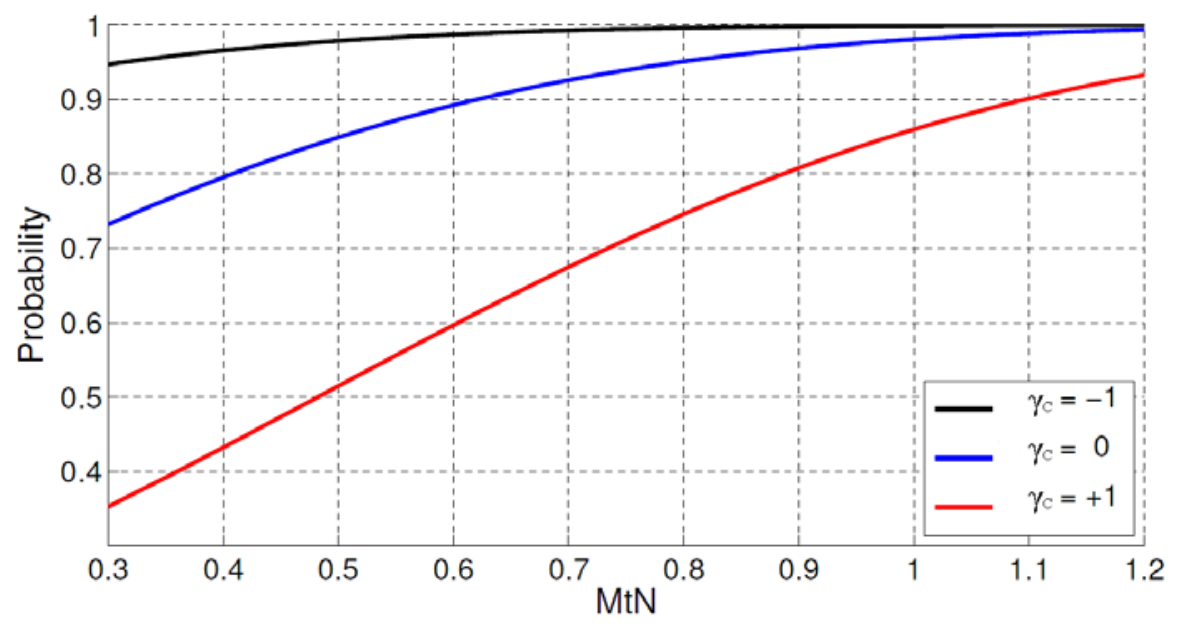

Fig. 15. Probability that the response with a filter of 3 px length is above the $\gamma_{C}$ parameter for three different cases.

\subsection{Parameter $\gamma_{M}$}


The $\gamma_{M}$ parameter is designed to exclude pixels where the $C$ value has fallen more than $\gamma_{M}$ below the maximum of the previous $C$ values. For a long streak ( $L=99 \mathrm{px}$ ) and a given $M t N$ value, the progression (using $\Lambda$ ) of the $C$ values for the central pixel is investigated over a sample of 1000 images and the probability of a decrease by a certain amount is determined. The results are shown in the left plot of Fig. 16. The right plot shows the distribution of all the occurrences of a decrease for $M t N=0.5$. The probability for a decrease is larger at lower $M t N$ values and a decrease of at least -0.5 occurs with a probability of less than 0.05 even for an $M t N$ of 0.5 . This justifies the choice of $\gamma_{M}=0.5$ as default value.
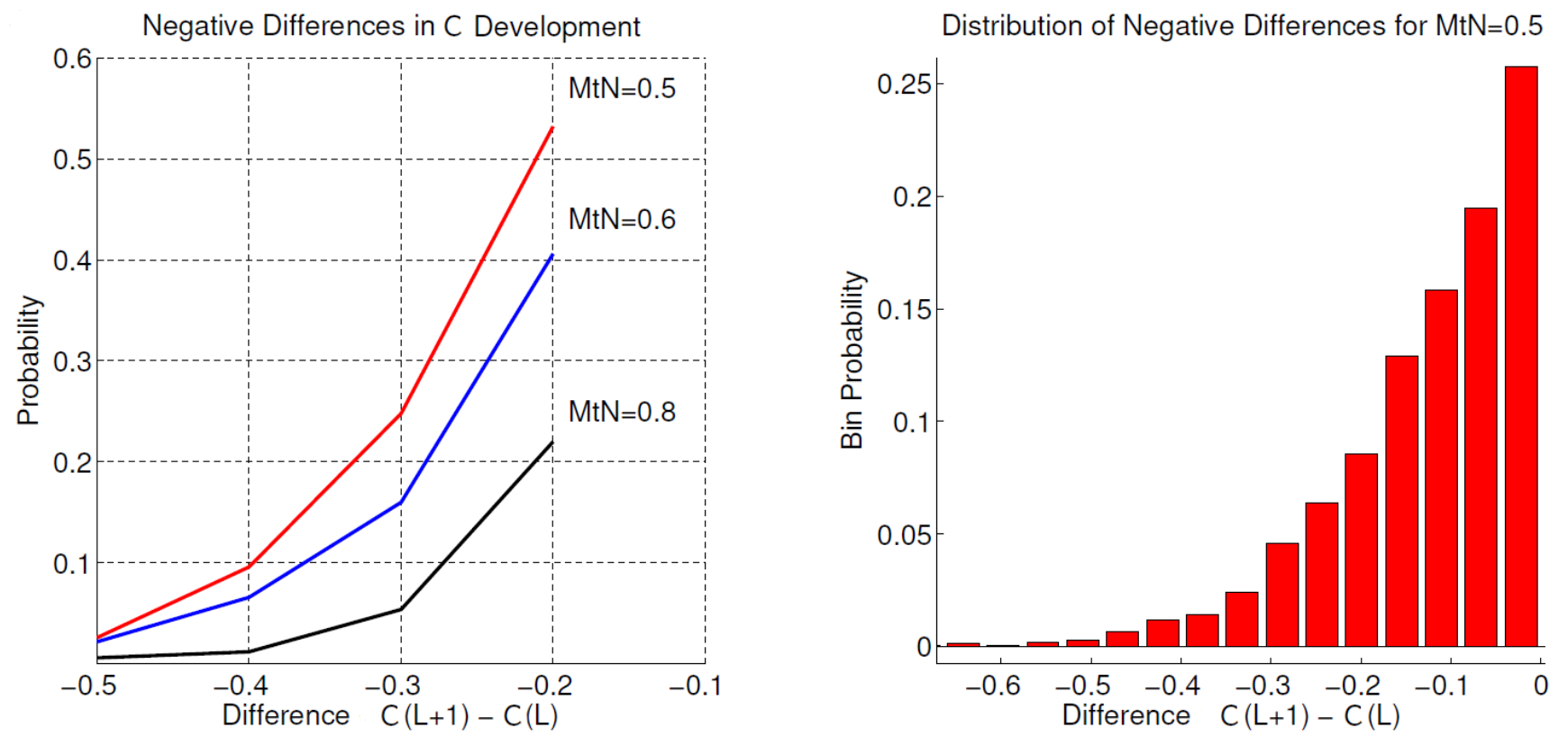

Fig. 16. Investigation of a streak of length $L=99$ px. Left plot: probability of a certain negative difference in $C$ of the central pixel. Right plot: distribution of negative differences in $C$ of the central pixel for an $M t N$ of 0.5 .

\subsection{Parameter $\gamma_{P}$}

The $\gamma_{P}$ parameter excludes pixels with low $P_{L}$ values from further investigation. To estimate the effect of this parameter, we simulate 1000 images with a streak of length $99 \mathrm{px}$ and a low $M t N$ value of 0.5 . The number $N$ of pixels where $P_{L}(99 \mathrm{px})$ has a greater value than a given $\gamma_{P}$ is determined. In Fig. 17 the probability that $N=0$ in the simulated distributions is shown. Thus the default setting of $\gamma_{P}=0.5$ leads to a probability for a total loss of faint streaks around 0.01 . This is low enough to guarantee a good detection sensitivity.

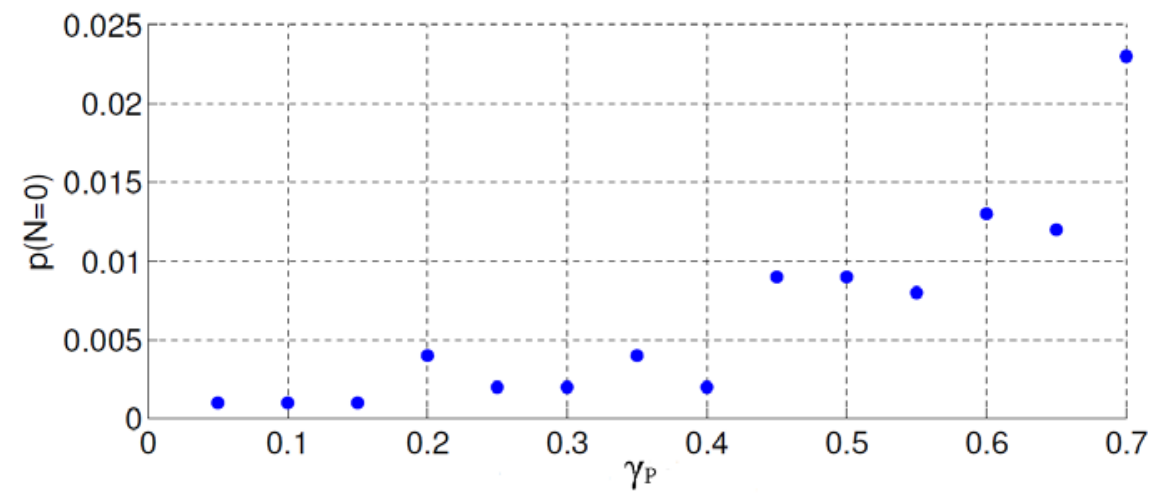

Fig. 17. Probability that no pixel of a streak of length $99 \mathrm{px}$ and $M t N=0.5$ survives the $\gamma_{P}$ parameter criterion.

\subsection{Parameter $\gamma_{R}$}


The choice of $\gamma_{R}$ in the angle restriction criterion is critical and should not be set too high in order not to lose long weak streaks. The detection probability in a sample of 1000 images for streaks of different lengths with $M t N=$ 0.6 is shown in Fig. 18. The values obtained applying the algorithm with and without $\gamma_{R}$ are compared with the ones from a single perfectly matching filter. The case with a setting $\gamma_{R}=0.995$ shows a slight flection in the curve with a reduction of about 0.1 in the probability for long streaks. However, we choose this setting as default since it can already provide a substantial improvement in computation time as shown in Fig. 9. Note that for streaks shorter than 50 px the detection probability in Fig. 18 of the tests is higher than in the perfect match. The reason is that in the tests also filters shorter than the streak length and/or with a slightly different angle than the streak angle can lead to an accepted detection.

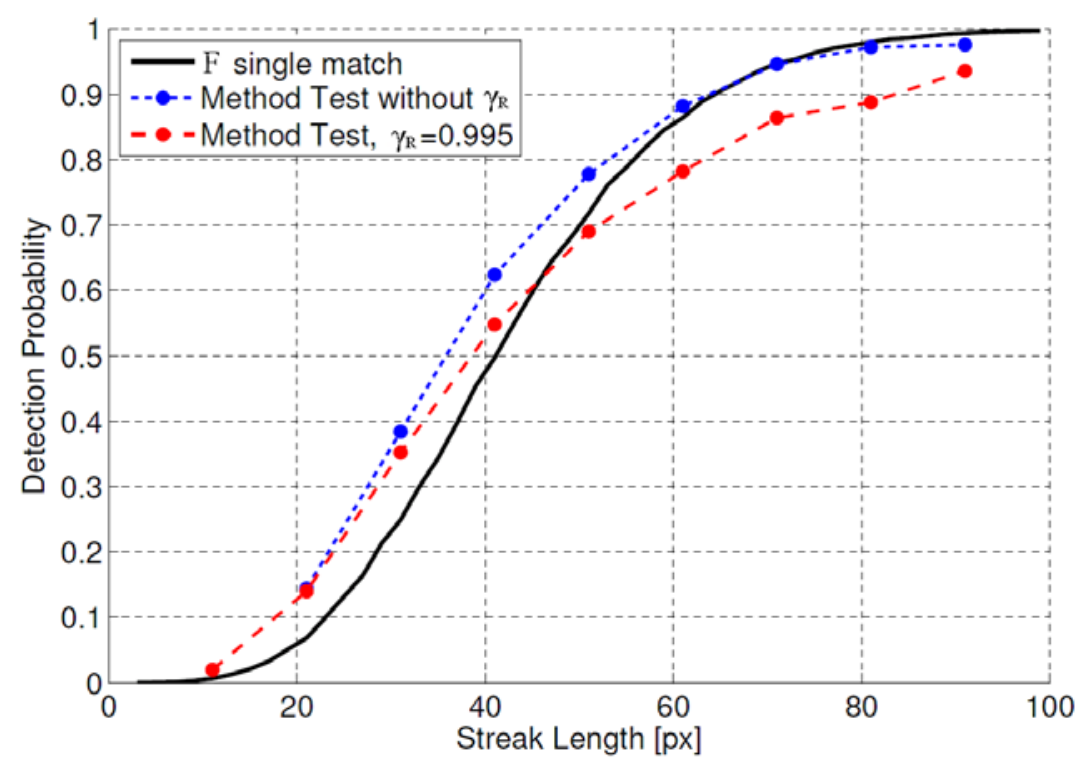

Fig. 18. Detection probabilities $(M t N=0.6)$ for: perfect match, algorithm without $\gamma_{R}$, and with $\gamma_{R}=0.995$.

\subsection{Computation time estimation}

The dependence of the processing time from the size of the image can be used to estimate the computational needs for the deployment of the detecting method. The algorithm with and without the angle restriction setting was applied to subframes of a real image with increasing size. The results are illustrated in Fig. 19. The computation time on the used system (2.5 GHz Intel Core i5, $8 \mathrm{~GB}, 1600 \mathrm{MHz}$ DDR3) with an implementation written in MATLAB can be extrapolated to 2-3 hours for a $4000 \times 4000$ pixels image.

The time increase is not just proportional to the square of $n$ as expected. Fitting a polynomial $f(n)$ of third degree to the data with angle restriction shows that there is a non-negligible third degree contribution:

$$
f(n)=4.2859-0.0257 n+2.5438 \cdot 10^{-4} n^{2}+8.0510 \cdot 10^{-8} n^{3} .
$$

This could be explained by the fact that the convolution for pixels close to the frame border needs less computation time, since the filter does not extend over the border, and trying to fit this behaviour introduces a third degree term. Another possibility is a non-linear behaviour of the MATLAB implementation or of the computing system due to e.g. CPU cache limitation or RAM shortage. 


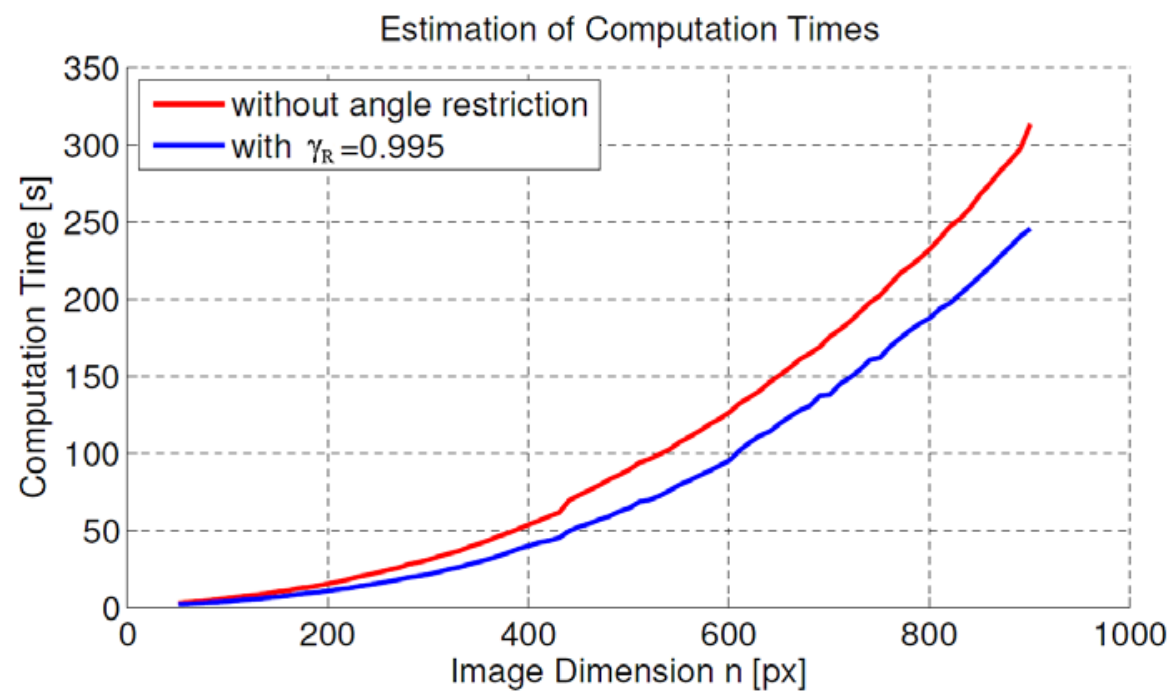

Fig. 19 Computation time depending on the dimension $n$ of a $(n \times n)$ image.

\section{Tests on real images}

The applicability of the detection algorithm was tested on real images. These were acquired by the ZimSMART telescope (Fujimoto et al., 2014) at the Observatory of the Astronomical Institute of the University of Bern, located in Zimmerwald. The images were taken with sidereal tracking, they contain streaks of artificial satellites with known positions in the sky and consequently with known coordinates on the acquired frames. This allows us to compare the algorithm detection with the ground truth.

Since the focus of this work is not on methods for background estimation and star removal, a simple procedure was applied for these two steps. In the background estimation step the statistics of small $10 \times 10 \mathrm{px}$ subframes covering the overall frame is evaluated fitting the histogram of the intensities in the subframes with a Gaussian function. The pixel values in the subframes are then corrected using the obtained values of mean and standard deviation. For the star removal, the frame regions with pixel values over a $4 \sigma$ threshold are selected (the standard deviation $\sigma$ refers to the overall frame). Within these regions the pixels surrounding the one with the maximal value are analysed and if on average in all directions the values similarly decrease, the region is considered a pointlike object or star and accordingly removed.

For each streak on the real frames the following characteristics were known:

- Approximate $\mathrm{x}$ and y coordinates of the center of the streak on the image.

- Approximate streak length in 3 categories: short (2 - 5 px), medium (30 - 50 px) and long (> $100 \mathrm{px})$.

- Estimated maximal pixel to noise ratio in 4 categories: high $(M t N \approx 5-10)$, medium $(M t N \approx 2-3)$, low (MtN $\leq 1)$.

- Degree of intensity variation along the streak in 3 categories: no significant variation, modest variation, discontinuous.

Out of 63 acquired frames with the original size of 4096 x 4096 px, we selected 28 subframes with a streak in the center, showing different examples of the above categories. For computation reasons a subframe size varying from 161 x 161 px up to 241 x 241 px was chosen. After applying the detection algorithm the $S_{F}$ image was inspected to see if $S_{F}$ indicates the detection of the streak and if the length, direction, and intensity in $S_{F}$ approximately corresponds to the streak characteristics on the frame. The selected number of test images is certainly not enough to provide a reasonable statistics to evaluate the performance of the method. However, these tests reveal to a certain extent the applicability of the algorithm to real cases. Table 2 summarizes according to the above categories the number of test images, detections, and false alarms.

\begin{tabular}{|c|c|c|c|c|c|c|c|c|}
\hline Streak length / light var. & \multicolumn{3}{|c|}{ short / no var. } & \multicolumn{3}{|c|}{ medium / no var. } & $\begin{array}{c}\text { long / no } \\
\text { var. }\end{array}$ & $\begin{array}{l}\text { long / } \\
\text { disc. }\end{array}$ \\
\hline $\mathrm{MtN}$ & low & med. & high & low & med. & high & lov & \\
\hline
\end{tabular}




\begin{tabular}{|l|c|c|c|c|c|c|c|c|c|c|}
\hline Num. images & 2 & 2 & 2 & 4 & 2 & 4 & 4 & 2 & 2 & 4 \\
\hline Num. detections & 2 & 2 & 2 & 4 & 2 & 4 & 4 & 2 & 2 & 4 \\
\hline False alarms (star removal) & 0 & 1 & 0 & 7 & 4 & 3 & 20 & 5 & 11 & 5 \\
\hline False alarms & 0 & 1 & 0 & 3 & 0 & 3 & 3 & 0 & 1 & 8 \\
\hline
\end{tabular}

Table 2: Summary of test images and detection results. The first row indicates the above categories of the streak length (short, medium, long) and the degree of intensity variation (no significant variation, modest variation, discontinuous). False alarms due to the star removal procedure are counted separately.

Since the declared values for the estimated $M t N$ are not close to the detection limits seen in simulated images, we expected to be able to detect most of the streaks in those real images. In fact, as we can see from the number of detections the proposed method was able to detect all of the investigated streaks. Note that the subframes contain only one streak and the number of detections is equal to the number of images in all the categories. As discussed in 3.1 the number of detections for a given $M t N$ depends on the choice of the threshold $b$, which in this case was low enough to guarantee the detection with high probability. Even in the difficult cases, expected for short streaks and low $M t N$, the detection was successful, although probably tests with more images, i.e. a better statistics, would show a reduced detection rate as expected. The intensity variation along the streaks and the presence of discontinuities did not affect the detection of long streaks. The total intensity over these long streaks is enough to compensate for some faint or missing parts of the streak and even if a discontinuity is present the separated parts are recognized as pieces of a unique streak since they are aligned.

The analysis of the $S_{F}$ images reveals also the presence of false alarms. A closer inspection shows that most of them are not due to the detection algorithm itself but are related to the star removal procedure. In fact the latter procedure is not always able to successfully remove diffraction patterns of bright stars, groups of adjacent stars, and very faint stars. These false alarms are indicated in a separate row in Table 2 . Besides these, few false alarms expected according to the detection statistics are also present. The number of these false alarms is in all cases equal or lower than the number of detections and of false alarms due to the star removal procedure, except in the category with long and discontinuous streaks. However, in this category for two frames it was difficult to identify the very faint stars and the given numbers are uncertain.

Fig. 20 to Fig. 24 illustrate examples of streaks with low $M t N$ and different lengths. In each case, the left image shows the frame after background estimation. To improve for the reader the visibility of faint structures the greyscale is set in the range between a lower limit of -5 and an upper limit of 5 . Values lower or greater than these limits are set to -5 or 5 , respectively. The middle image shows the frame after star removal, with limits of -3 and 3. The right image shows in logarithmic scale the content of $S_{F}$ which indicates the geometry of the accepted filters and therefore the position of the detected object. The expected detections are in the middle of the frame, while any additional detection corresponds to a false alarm or to an unknown real object.
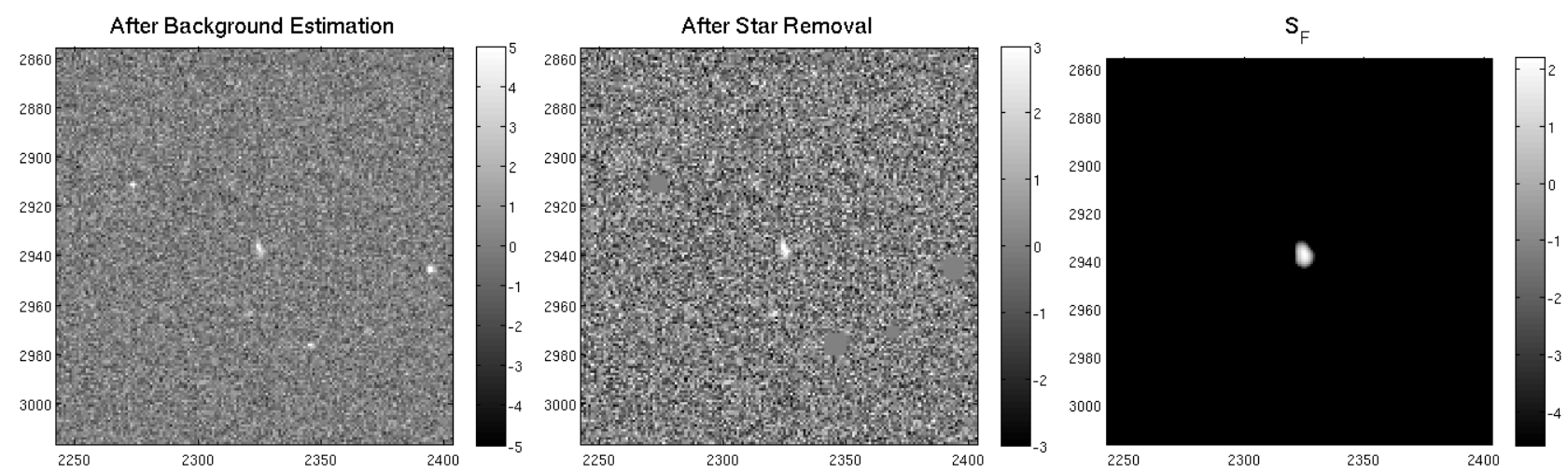

Fig. 20. Frame with short streak and $M t N \sim 1$. 

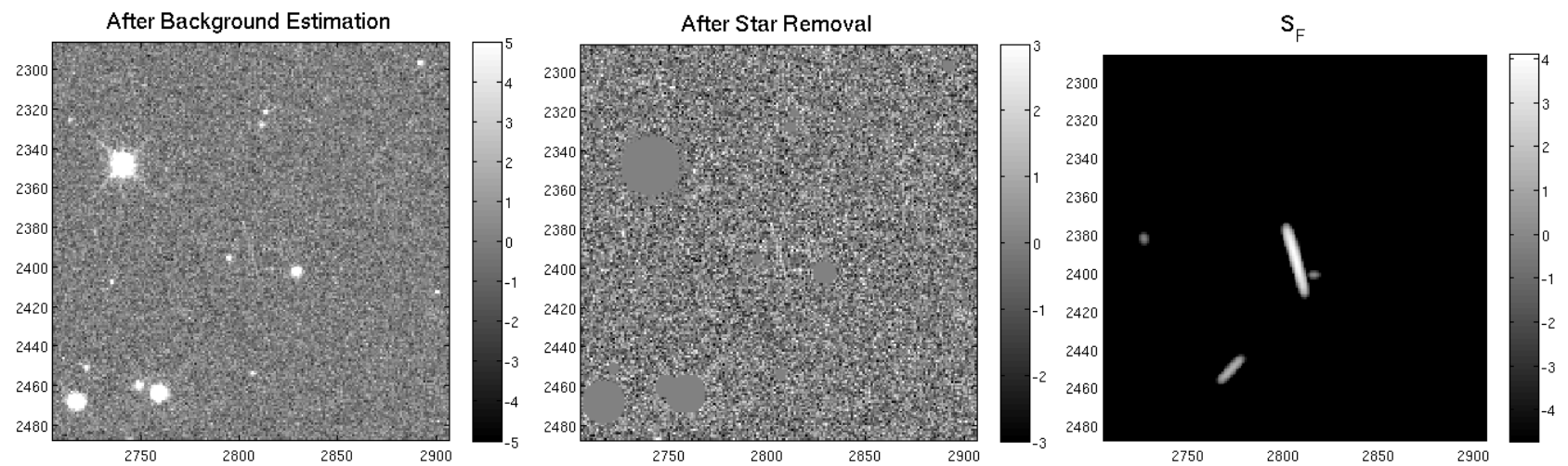

Fig. 21. Frame with streak of medium length $(\sim 50 \mathrm{px})$ and $M t N \sim 1$. The shorter detected streak is probably a false alarm due to the diffraction spike of one of the bright stars, while the two point-like detections are false alarms due to not removed stars.
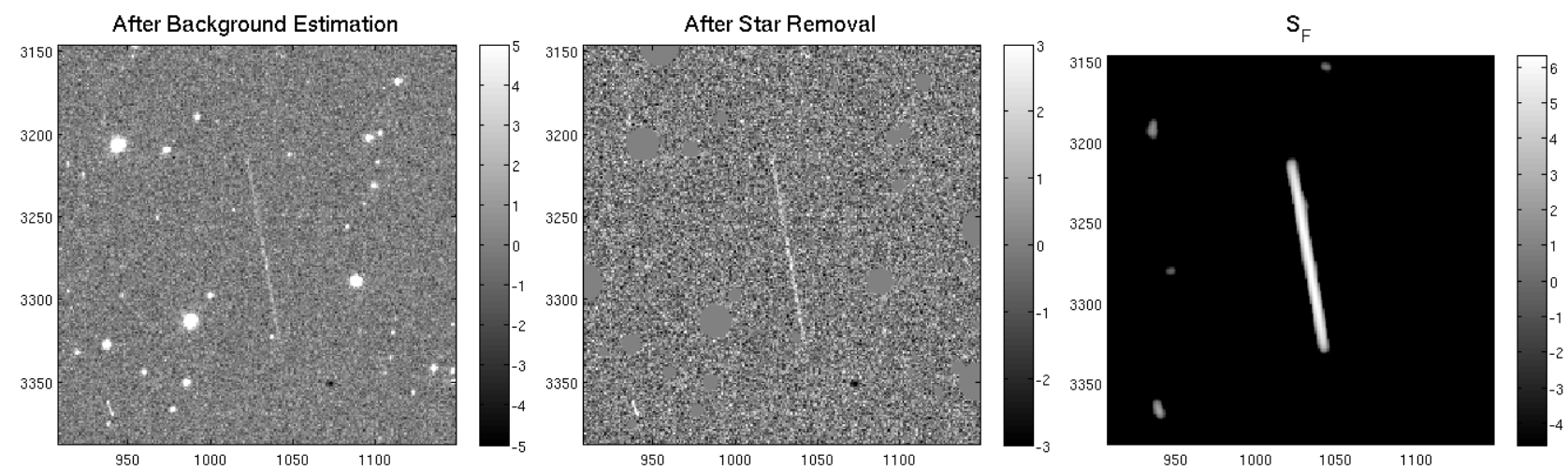

Fig. 22. Frame with long streak ( $100 \mathrm{px})$ and $M t N \sim 1$. The detection in the lower left corner possibly corresponds to an unexpected real object, while the other shorter ones are false alarms due to not removed stars.
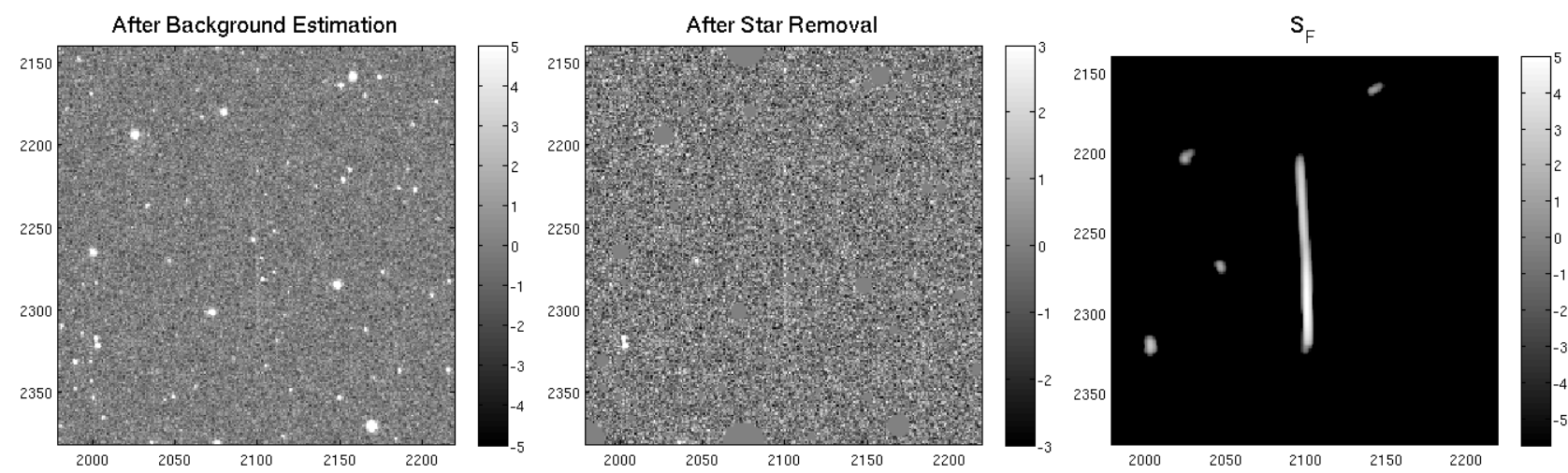

Fig. 23. Frame with long streak $(\sim 100 \mathrm{px})$ and $M t N \sim 0.5$. The detection in the lower left corner is due to a double star, while the other short ones are false alarms due to not removed stars. 

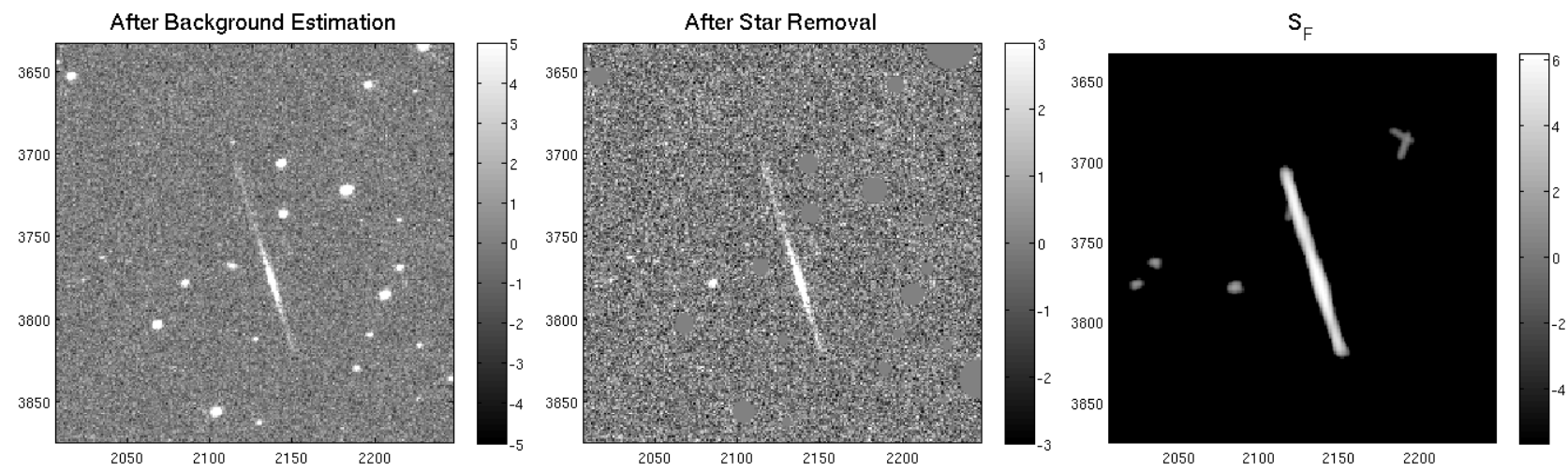

Fig. 24. Frame with long streak ( $100 \mathrm{px})$ and $M t N \sim 1$. The streak is discontinuous. The point-like detections are false alarms due to not removed stars.

\section{Conclusions}

A method is proposed to detect streaks on CCD frames using a streak-like spatial filter. The idea is to use filters matching the expected shape of the region to be detected. The original frame is convolved with streak-like filters of different lengths and orientations. A threshold is then used to accept or reject regions of interest according to the convolution values. The filtering process selectively improves the signal of streak-like regions and provides frames which contain in the end clear detected streaks. From these detected regions the exact characteristics of the streak, e.g. centroid and length, can be extracted with further processing, but this part is not analysed in this work. The detection probability depends on the length and on the $M t N$ of the streak. Especially for long streaks, a detection for even very low $M t N$ values (below 0.5 ) is possible. These low values confirm somehow the expectations, since from a theoretical point of view a method based on a filter perfectly matching the shape, length and orientation of a streak should lead to the best detection sensitivity.

Since a number of artefacts is present in real images, additional procedures are necessary which involve further algorithm parameters. We have tried to justify the settings of the algorithm parameters with statistical arguments. Most of the optimal values do not depend on the length, orientation, or $M t N$ of the streak. The only parameter which is set according to the needs of the user is the threshold $\mathrm{b}$, while the clipping parameter $c(L, x, y)$ might be tuned as a function of the streak length. The parameters are based on the theoretical detection probability of a perfect matching filter and are chosen in such a way to be optimal in a statistical sense for different detection conditions. Therefore, the suggested default values do not need to be adapted as a function of the characteristics of the acquired frame. However, the parameters have interdependencies, e.g. the choice of lengths in $\Lambda$ influences the quality of the length limitation criterion. Obviously, these interdependencies reduce their general validity. Also, the filter is in general not a perfect matching filter and the theoretical probability is only an approximation.

To evaluate the applicability to real cases the method was applied to subframes with reduced size of real acquired CCD images. Images of streaks with different lengths (from 2 - 5 px up to more than $100 \mathrm{px}$ ), MtN values (from 1 up to 10), and intensity variations or discontinuities, were selected.

The proposed method was able to detect all of the investigated streaks as expected according to the choice of the detection threshold. Even intensity variations or discontinuities along the streaks did not affect the detection of long streaks. A number of false alarms was present in the results. However, most of these turned out to be due to a not very sophisticated star removal procedure, which did not remove diffraction patterns of bright stars, groups of adjacent stars, and very faint stars. The remaining false alarms were in general fewer than the correct detections for the selected detection threshold.

Compared to stacking techniques, which are among the most sensitive methods, this algorithm has the advantage of less complex requirements in terms of image acquisition process and computing hardware. On the other hand, image stacking has the advantage of an improved signal-to-noise ratio. Both methods have basically the same intent. While the stacking methods try to collect the pixels of interest from different images, our method tries to collect them by extending and moving the collecting area (or filter) on one image. Since in both cases the principle is similar the detection sensitivity is expected to be approximately in the same range. The proposed approach has close similarity with the method in (Levesque, 2009). In the latter the same collection principle with a streak 
matching filter is suggested. However, in our proposed algorithm the streak matching idea is applied to all possible orientations and lengths and additional artefacts are addressed.

For the tests all real images were acquired with sidereal tracking and the stars on the images appear more or less as point-like objects. If the images are acquired using another tracking mode, e.g. object tracking, then the stars might also appear as streaks. In this case one of the difficulties is to distinguish the object streak from the star streaks. Usually one possibility is to distinguish the star streaks based on their length and preferential direction, which are different than the ones of the object streak. If this information is known it is possible to use the current algorithm with restricted sets $A$ and $\Lambda$, that contain only the required angles, respectively lengths, to detect and subsequently remove the star streaks. In the successive step the algorithm is applied with another angle and length restriction to detect the object streak. In general a consequence of not having stars as point-like objects on the image is a higher contamination of the star streaks in the filtering process which affects the sensitivity of the method. Tests on images acquired in other tracking modes have not been performed yet, but in future work it might be interesting to evaluate the current method applied to these images.

As already mentioned the purpose of the proposed method is the detection of the streak and not its characterization in terms of centroid, length, brightness. The present method yields the accepted filter responses in the quantities $S_{C}$ and $S_{F}$, which still do not contain explicitly the location, strength, and geometrical dimension of the detected streak. Here the detections are considered as object candidates where the centroiding procedure or a more detailed investigation of orientation and length has still to be performed. A future task will be the further processing of $S_{C}$ and $S_{F}$ to extract the necessary information. The values in $S_{C}$ and $S_{F}$ can be a basis for a centroiding criterion since they indicate the number and weight of superimposed accepted convolution values. The fact that the area density of nonzero pixels in $S_{C}$ is higher in bright and well defined streaks can be exploited.

Since the capacity of detection mainly depends on the statistics of the image background it is crucial to have a background estimation, prior to the detection process, as accurate as possible. This part was not addressed in this work and needs to be taken into account for further improvements. Most of the detections which do not belong to objects of interest, are due to remaining stars or other non-background structures as cosmics or diffraction patterns of stars. Removing such artefacts in advance of the detection should save time in the evaluation of the results after the convolution. The used star removal method is very simple and does not address e.g. the recognition of cosmics. Also this part needs to be further investigated.

The computation time on the used system and implemented in MATLAB is in the range of 2-3 hours for a $4000 \mathrm{x}$ 4000 pixels image. The angle restriction procedure is one attempt to include in the detection method a criterion to save computation time. In particular situations where the length and orientation of streaks is approximately known the set $A$ and $\Lambda$ can be restricted, as previously explained. The angle restriction could possibly be extended to an area restriction where, with increasing length, only areas of interest are further considered. The parallelization of part of the algorithm has also to be analysed as well as the possibility to improve the speed of the process by using GPUs.

\section{References}

Ciurte, A., Danescu, R., 2014. Automatic Detection of MEO Satellite Streaks from Single Long Exposure Astronomic Images. In: Proceedings of the $9^{\text {th }}$ International Conference on Computer Vision Theory and Applications, Lisbon, Portugal.

Dao, P., Crabtree, P., McNicholl, P., 2013. Blind Search of Faint Moving Objects in 3D Data Sets. In: Proceedings of the Advanced Maui Optical and Space Surveillance Technologies Conference, Maui, Hawaii, USA.

Fujimoto, K., Scheeres, D., Herzog, J., Schildknecht, T., 2014. Association of optical tracklets from a geosynchronous belt survey via the direct Bayesian admissible region approach. Advances in Space Research 53, 295 - 308.

Herzog, J., Schildknecht, T., Hinze, A., Ploner, M., Vananti, A., 2013. Space Surveillance Observations at the AIUB Zimmerwald Observatory. In: Proceedings of the $6^{\text {th }}$ European Conference on Space Debris, Darmstadt, Germany.

Johnson, N.L., Kotz, S., Balakrishnan, N. (1994). Continuous Univariate Distributions, Vol. 1. New York: John Wiley \& Sons, Inc.

Levesque, M.P., 2009. Automatic Reacquisition of Satellite Positions by Detecting Their Expected Streaks in Astronomical Images. In: Proceedings of the Advanced Maui Optical and Space Surveillance Technologies Conference, Maui, Hawaii, USA.

Sara, R., Matousek, M., Franc, V., 2013. RANSACing Optical Image Sequences for GEO and near-GEO Objects. In: Proceedings of the Advanced Maui Optical and Space Surveillance Technologies Conference, Maui, Hawaii, USA. 
Sustr, M. (2013). Streak Detection in Astronomical Images. Research Reports of CMP, Czech Technical University in Prague, No. 11.

Torteeka, P., Gao, P., Shen, M., Guo, X., Yang, D., Yu, H., Zhou, W., Tong, L., Zhao, Y., 2019. Autonomous space target tracking through state estimation techniques via ground-based passive optical telescope. Advances in Space Research 63, 461 -475 .

Virtanen, J., Poikonen, J., Säntti, T., Komulainen, T., Torppa, J., Granvik, M., Muinonen, K., Pentikäinen, H., Martikainen, J., Näränen, J., Lehti, J., Flohrer, T., 2016. Streak detection and analysis pipeline for space-debris optical images. Advances in Space Research 57, 1607 - 1623.

Yanagisawa, T., Nakajima, A., Kimura, T., 2003. The Stacking Method: the technique to detect small size of GEO debris. Science and Technology Series 109, American Astronautical Society, Univelt, Inc.

Yanagisawa, T., Nakajima, A., 2005. Detection of Small LEO Debris with Line Detection Method. Transactions of the Japan Society of Aeronautical Space Sciences 47(158), 240 - 248.

Yanagisawa, T., Kurosaki, H., Banno, H., Kitazawa, Y., Uetsuhara, M., Hanada, T., 2012. Comparison between four Detection Algorithms for GEO Objects. In: Proceedings of the Advanced Maui Optical and Space Surveillance Technologies Conference, Maui, Hawaii, USA.

Zimmer, P.C., Ackermann, M.R., McGraw, J.T., 2013. GPU-accelerated Faint Streak Detection for Uncued Surveillance of LEO. In: Proceedings of the Advanced Maui Optical and Space Surveillance Technologies Conference, Maui, Hawaii, USA. 